\title{
Epidural Electrical Stimulation Of The Cervical Dorsal Roots Restores Voluntary Arm Control In Paralyzed Monkeys
}

\section{Marco Capogrosso (D MCAPO@pitt.edu ) \\ University of Pittsburgh}

\section{Beatrice Barra}

University of Fribourg

\section{Sara Conti}

University of Fribourg

\section{Matthew Perich}

Icahn School of Medicine at Mount Sinai https://orcid.org/0000-0001-9800-2386

\section{Katie Zhuang}

University of Fribourg

\section{Giuseppe Schiavone}

Ecole Polytechnique Federale de Lausanne

\section{Florian Fallegger}

Ecole Polytechnique Federale de Lausanne

\section{Katia Galan}

Ecole Polytechnique Federale de Lausanne

Nicholas James

EPFL

\section{Quentin Barraud}

Swiss Federal Institute Of Technology (EPFL) https://orcid.org/0000-0003-0894-1959

\section{Maude Delacombaz}

University of Fribourg

\section{Mélanie Kaeser}

Dept. of Medicine University of Fribourg

\section{Eric Rouiller}

Dept. of Medicine University of Fribourg

\section{Tomislav Milekovic}

Ecole Polytechnique Federale de Lausanne

\section{Stephanie Lacour}

Ecole Polytechnique Fédérale de Lausanne (EPFL) https://orcid.org/0000-0001-9075-4022 Jocelyne Bloch 
University Hospital of Lausanne

\section{Gregoire Courtine}

École Polytechnique Fédérale de Lausanne https://orcid.org/0000-0002-5744-4142

\section{Article}

Keywords: cervical dorsal roots, paralysis, voluntary arm control

Posted Date: April 19th, 2021

DOl: https://doi.org/10.21203/rs.3.rs-395156/v1

License: (c) (i) This work is licensed under a Creative Commons Attribution 4.0 International License. Read Full License

Version of Record: A version of this preprint was published at Nature Neuroscience on June 30th, 2022. See the published version at https://doi.org/10.1038/s41593-022-01106-5. 


\section{EPIDURAL ELECTRICAL STIMULATION OF THE CERVICAL DORSAL ROOTS RESTORES VOLUNTARY ARM CONTROL IN PARALYZED MONKEYS}

3 B. Barra ${ }^{1,2, *}$, S. Conti ${ }^{1, *}$, M.G. Perich ${ }^{3}$, K. Zhuang', G. Schiavone ${ }^{4}$, F. Fallegger ${ }^{4}$, K. Galan ${ }^{5,7}$, N. 4 D. James ${ }^{5}$, Q. Barraud 5,7, M. Delacombaz, ${ }^{1,7}$, M. Kaeser ${ }^{1}$, E. M. Rouiller ${ }^{1}$, T. Milekovic ${ }^{3,7}$, S. Lacour $^{4}$, J. Bloch 6,7 , G. Courtine ${ }^{5,6,7}$ and M. Capogrosso ${ }^{1,2,8}$

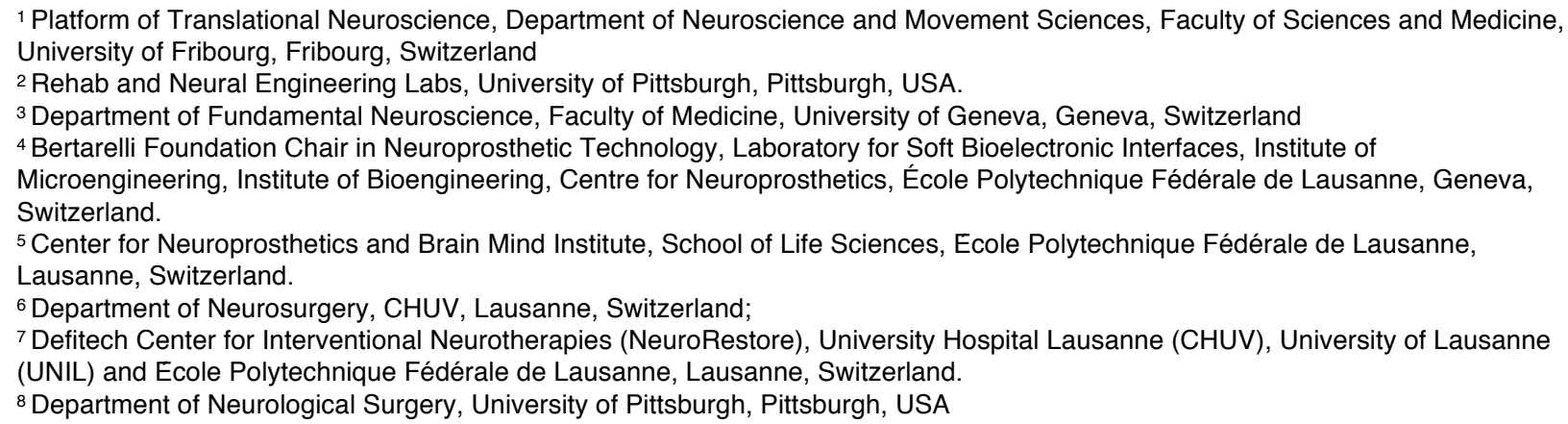

*these authors contributed equally to this work

20 Correspondence to: Marco Capogrosso - mcapo@pitt.edu

\section{SUMMARY}

Recovering arm control is a top priority for people with paralysis. Unfortunately, the complexity of the neural mechanisms underlying arm control practically limited the effectiveness of neurotechnology approaches. Here, we exploited the neural function of surviving spinal circuits to restore voluntary arm and hand control in three monkeys with spinal cord injury using spinal cord stimulation. Our neural interface leverages the functional organization of the dorsal roots to convey artificial excitation via electrical stimulation to relevant spinal segments at appropriate movement phases. Stimulation bursts, triggered by intracortical signals produced sustained arm movements enabling monkeys with arm paralysis to perform an unconstrained, three-dimensional reach-and-grasp task. Stimulation specifically improved strength, task performances and movement quality. Electrophysiology suggested that artificial recruitment of the sensory afferents was synergistically integrated with spared descending inputs and spinal reflexes to produce coordinated movements. The efficacy and reliability of our approach hold realistic promises of clinical translation. 
40 More than 5 million people in the US currently live with some form of motor paralysis ${ }^{1}$. Stroke and spinal cord injury $(\mathrm{SCl})$ are the main causes with hundreds of thousands of new cases per year². Impairments of the hand and arm are particularly problematic, representing a major unmet need for both $\mathrm{SCl}$ and stroke patient populations $\mathrm{s}^{3,4}$. Indeed, even mild deficits in hand function lead to significant degradation of quality of life. Unfortunately, recovery of hand and arm motor function is still an unsolved clinical challenge.

Generated in the cerebral cortex, upper limb motor commands are relayed to subcortical and spinal circuits that activate motoneurons and regulate sensory inputs to produce skilled motor actions $^{5-8}$. Spinal cord injury (SCl), or stroke, damage these communication pathways generating impairments in sensory regulation and motor functions that lead to motor paralysis.

Historically, neurotechnologies were conceived around the idea of restoring movements in paralyzed subjects via a technological bypass. Such solution would use signals from cortical areas as inputs and artificially compensate for lack of motoneuron activation by producing desired muscle activity below the lesion' ${ }^{9}$. For example, functional electrical stimulation (FES) was used to activate arm muscles in response to intracortical neural activity from the motor cortex ${ }^{10,11}$. This pioneering concept allowed paralyzed monkeys and humans to perform voluntary grasping tasks ${ }^{10-13}$. However, translation of these concepts into daily clinical practice is hindered by two distinct limitations. First, the artificial motoneuron recruitment order generated by FES induces muscle fatigue ${ }^{14}$ which is particularly problematic for arm movements. Indeed, fatigue prevents the generation of sustained forces and consequently FES fails to enable sustained threedimensional arm movements that are required for daily activities. Second, since FES bypasses surviving circuits in the spinal cord, complex stimulation protocols ${ }^{15}$ and sophisticated decoding algorithms ${ }^{10,13}$ are required to orchestrate the activation of multiple muscles and produce functional movements. As a result, these systems require an articulated combination of hardware and software. Unfortunately, this complexity does not cope well with dynamic clinical environments that need robust and practical solutions for a rapid set up and large-scale use.

In contrast, epidural electrical stimulation (EES) of the lumbar spinal cord exploits surviving spinal circuits and supra-spinal connections after injury to produce movements ${ }^{16}$. Similar to intraspinal stimulation ${ }^{17-19}$, EES engages motoneurons via direct recruitment of large sensory afferents ${ }^{20,21}$ leading to widespread excitatory post-synaptic potentials in the spinal cord. More importantly, since motoneurons are recruited via natural synaptic inputs, EES generates a natural recruitment order ${ }^{22,23}$ that is resistant to artificial fatigue. This enables the production of forces that can sustain the whole-body weight ${ }^{24}$. Moreover, engagement of motoneurons from pre-synaptic pathways allows residual descending inputs and spinal circuits to control motoneurons excitability and produce voluntary movement after complete motor paralysis ${ }^{25,26}$.

Building on animal models $27-29$, recent clinical studies have shown that continuous stimulation delivered through epidural implants on the dorsal aspect of the lumbosacral spinal cord increased muscle strength, voluntary muscle activation and single joint movements in people with complete leg paralysis ${ }^{26,30,31}$. More strikingly, when coupled with targeted physical rehabilitation protocols, continuous EES restored weight bearing locomotion in subjects with severe $\mathrm{SCl}^{32,33}$. These outstanding clinical results prompted experimental studies aiming at verifying whether EES could be used to promote also upper limb movements after $\mathrm{SCl}^{34}$. Unfortunately, while clinical studies showed some success in improving hand grip force with both epidural and non-invasive approaches ${ }^{35,36}$, continuous EES did not produce results of similar outstanding efficacy as those 
observed for the lower limbs ${ }^{32,33}$. In fact, clinical outcomes were similar to those obtained with surface FES ${ }^{37}$.

Reasons for this discrepancy may stem from the complexity of upper limb motor control and biomechanics compared to locomotion. Indeed, in contrast to pattern-driven ${ }^{38,39}$ and repetitive locomotor movements, upper limb movements are composed by a non-repetitive and taskdependent combination of movement modules which are highly dependent from sophisticated cortico-spinal control $7,40-44$ and accurate sensory feedback ${ }^{42,45-47}$. Because of this intrinsic complexity, non-specific neuromodulation could limit the efficacy of EES by exciting all spinal segments simultaneously, irrespectively of movement phase. More importantly, unspecific and continuous stimulation of the sensory afferents through EES disrupts natural sensory inputs ${ }^{23}$ thus hindering spinal regulation of movements which is critical in dexterous upper limb control ${ }^{45-}$ 47.

99 We and others have shown that it is possible to direct electrical stimulation of the spinal cord to 100 target restricted segments during appropriate times $17,48,49$. These spatio-temporal stimulation 101 protocols enabled voluntary locomotion in monkeys with $\mathrm{SCl}$ as early as day 6 post injury without any physical training ${ }^{50}$ and within 2 weeks post implantation in humans with complete leg paralysis ${ }^{51}$. This approach exploits the somato-topography of the spinal sensory system to selectively engage restricted spinal regions ${ }^{21,49}$. Unfortunately, non-invasive technologies and clinically approved electrodes are unfit for this scope ${ }^{52,53}$ because of their limits in selectivity. Therefore, we hypothesized that a neural interface, specifically designed to target the cervical dorsal roots, could enable the administration of spatio-temporal stimulation patterns to the cervical spinal cord. We tested this hypothesis in three monkeys with a unilateral cervical SCl. We designed a personalized epidural interface to target primary afferents within the cervical dorsal roots. We hypothesized that the electrical stimulation of the roots with bursts linked to movement attempts would enable voluntary motor control and improve functional deficits of the arm and hand that emerge after SCl. Specifically we tested for improvements in muscle strength, dexterity and ability to execute three-dimensional functional tasks in full independence. Finally, we verified that the mechanisms enabling the voluntary recruitment of motoneurons in the cervical spinal cord were similar to those occurring during EES of the lumbosacral circuits.

\section{Results}

\section{Natural arm movements}

Clinically effective systems should enable truly functional arm movements rather than simplified tasks such as single-joint movements. A functional arm movement entails a coordinated activation of arm muscles to achieve a desired movement while supporting the arm weight at all times. Most of daily activities require arm extension (reach) and flexion (pull), combined with a hand-grasp without a constrained timing or structure. Consequently, we developed a robotic platform allowing the quantification of reach, grasp and pull movements ${ }^{54}$ that would feel natural and unconstrained to monkeys both in trajectory and timings (Figure 1A). We trained three adult Macaca fascicularis monkeys to reach for, grasp, and pull an instrumented object placed on the end effector of our robotic arm (Figure 1B). Movement trajectories were not constrained neither kinematically nor in time. Monkeys waited for the go signal, reached for the object and pulled to receive a food or juice reward when the object crossed a pre-defined displacement threshold ${ }^{54}$. Monkeys intuitively and rapidly ${ }^{29,30}$ learned this task by developing their own individual kinematic strategies (Extended Data Figure 1) and personal movement speeds. We then designed a battery of electrophysiology and kinematic measurements to evaluate functional outcomes on task performances, muscle 


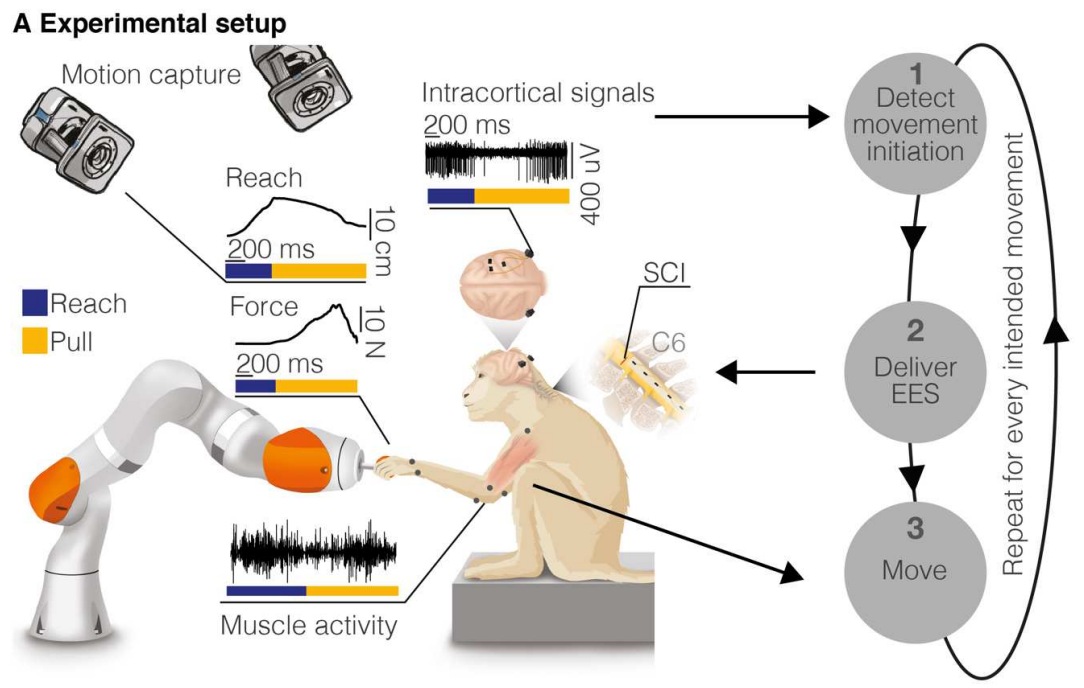

B Behavioral task

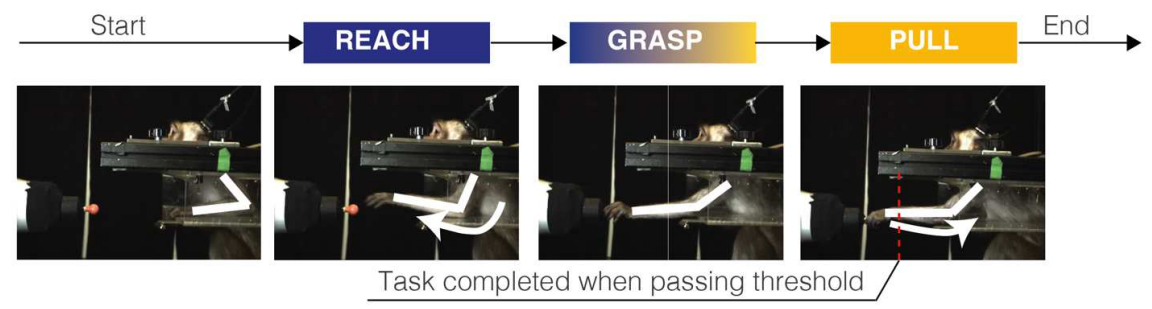

Figure 1. Experimental framework. (A) On the left, schematic of the behavioral experimental platform. While the animals were performing a robotic reach, grasp and pull task, we measured 3D forces applied to the robot joints, full-limb kinematics, electromyographic (EMG) activity from eight muscles of the arm and hand, and intra-cortical signals from sensorimotor areas. On the right, conceptual scheme of the experimental protocol: (1) A decoder running on a control computer identified movement attempts and (2) delivered electrical spinal cord stimulations to the appropriate spinal roots. (3) Stimulations produced arm and hand movement that we recorded and analyzed off-line. (B) Schematic illustration of the task. Monkeys were trained to reach for, grasp, and pull a target object placed at the end effector of a robotic arm. We considered a movement complete when a target spatial threshold was crossed during pull. Copyright Jemère Ruby.

activation, muscle strength and movement dexterity. Specifically, we quantified full-limb 3D kinematics (Vicon Motion Systems, Oxford, UK), pulling forces, and electromyographic (EMG) signals from intramuscular leads in eight arm muscles (Figure 1A). Before SCI, we observed clear bursts of EMG activity from all hand and arm muscles during the three movement phases: reach, grasp, and pull in all monkeys. Finally, to document the involvement of cortical neurons during movement enabled by EES and to extract signals that could be used to link stimulation bursts to movement phase onset, we implanted multi-microelectrode arrays (Blackrock Microsystems, Salt Lake City, USA) in the arm/hand region of the right sensorimotor (M1, S1) and ventral premotor (PMv) cortex. We validated these recordings by verifying that neural activity was consistently modulated with kinematics pre-injury and with the three movement phases as largely expected $^{54}$ (Figure 1, Extended Data Figure 1). In summary, we analyzed natural arm movements in monkeys and found that electromyographic and cortical activity were strongly modulated during the three different task phases. We concluded that in order for stimulation protocols to be effective, it was important to support these three phases independently. 


\section{Personalized spinal interface}

150 To design an optimal interface, we studied the anatomy of the monkey cervical spinal cord. We 151 extrapolated available anatomical information from literature and found that, similar to humans, 152 motoneurons innervating arm muscles in the monkeys are segmentally organized ${ }^{55}$ (Figure 2A). 153 We previously showed that stimulation of a single cervical dorsal root will recruit motoneurons that receive direct afferent inputs from that $\operatorname{root}^{53}$. Exploiting this property allows to obtain a segmental recruitment order of motoneurons that can be targeted to promote specific movement phases $49,51,56$. Therefore, we designed a spinal interface that could target each root independently. We achieved this by placing contacts on the lateral aspect of the cord to target the entry zone of each individual $\operatorname{roo}^{53}$. Since each monkey displayed a unique anatomy, we tailored the design of our interface to each specific subject. For this, we measured white matter diameter and vertebral canal features from computed tomography (CT) and magnetic resonance imaging (MRI). We then spaced the electrodes rostro-caudally and medio-laterally to match the transversal and longitudinal dimensions of the cord of each animal (Figure 2B, Extended Data Figure 2A). This allowed us to simplify the neural interface architecture by minimizing the number of contacts while maintaining high muscle recruitment specificity ${ }^{57}$. We then designed a surgical strategy to position the epidural interface between the $\mathrm{C} 6$ and $\mathrm{T} 1$ dorsal roots (Figure $2 \mathrm{C}$ ). We performed

A

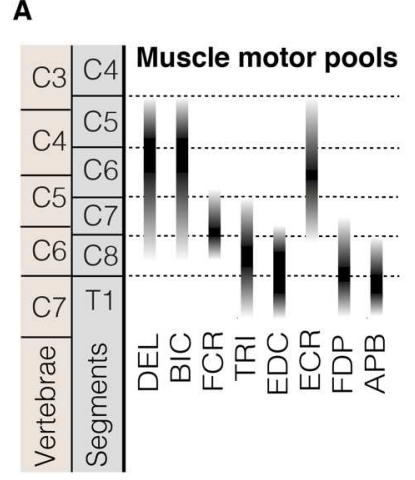

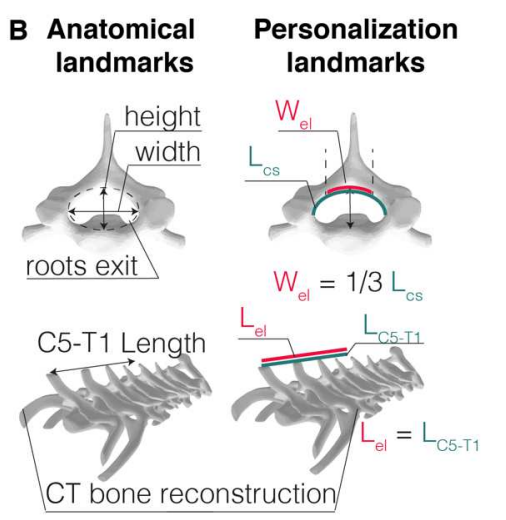

E
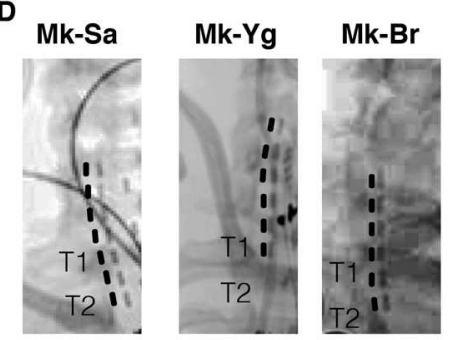
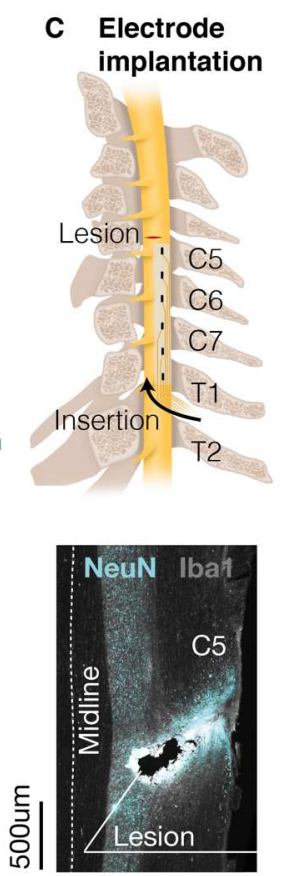

Figure 2. Epidural electrode design and implantation. (A) Motoneurons pool distribution of arm and hand muscles in the cervical spinal cord in relation to vertebrae and spinal segments (adapted from Jenny and Inukai, 1983). Deltoid (DEL), Biceps Brachii (BIC), Flexor Carpi Radialis (FCR), Triceps Brachii (TRI), Extensor Digitorium Communis (EDC), Extensor Carpi Radialis (ECR), Flexor Digitorium Profundis (FDP), Abductor Pollicis Brevis (ABP). (B) Anatomical landmarks used to tailor the epidural interface to each monkey's anatomy (Length of dorsal aspect of spinal canal Los, length of C5-T1 spinal segment Lc5-T1, electrode width $\mathrm{Wel}_{\mathrm{el}}$ electrode length Lel). Three-dimensional reconstructions of vertebras are obtained by CT-reconstruction (Osirix, Pixmeo, Switzerland). (C) Schematic representation illustrating the positioning and insertion of the spinal implant in the epidural space (D) Representative Xray scans of the epidural implant in the three monkeys (Mk-Sa, Mk-Br and Mk-Yg). (E) Anatomical reconstruction of the cervical spinal cord lesion (black area) for the 3 monkeys, shown on a transversal section (the percentage indicates the portion of the total spinal cord area that was injured on this transversal plane). On the right, representative image of longitudinal section of the spinal cord of $\mathrm{Mk}-\mathrm{Br}$ around the lesion site stained with NeuN (neuronal cell bodies) and Iba1 (microglia). 
laminectomies between the T1 and T2 vertebrae and the C5 and C6 vertebrae, then pulled the neural interface through the intermediate epidural space with the help of a custom soft inserter ${ }^{57}$. We verified that the position of the array remained stable for the entire duration of the study (up to 3 weeks) through repeated X-ray imaging (Figure 2D, Extended Data Figure 2B). During the same surgery, we performed a unilateral spinal cord injury at the C5/C6 segments (Figure 2E) aiming at transecting the cortico-spinal tract that is located on the lateral aspect of the white matter in monkeys. This type of lesion is amply described in literature and induces unilateral arm and hand paralysis 58,59 while preserving important bodily functions such as bladder control. Postmortem immunohistochemistry analysis of the spinal cords showed that the spinal interface did not damage the cervical cord in any of the three monkeys but did reveal that $\mathrm{Mk}-\mathrm{Br}$ received an unplanned compression injury at the insertion site (T3 spinal segment). Given the caudal position of this contusion it is likely for it to have occurred during implantation (Extended Data Figure 2C). Since the T3 segment is below the innervation of the arm motoneurons, this lesion did not affect the phenotype of arm and hand motor deficits which did not differ from the other monkeys (see Methods).

181 In summary, we designed a spinal interface to selectively recruit the cervical dorsal roots. We tailored the interface to the specific anatomy of each monkey and designed a surgical strategy to perform a consistent and stable implantation.

A Muscle recruitment during single pulse

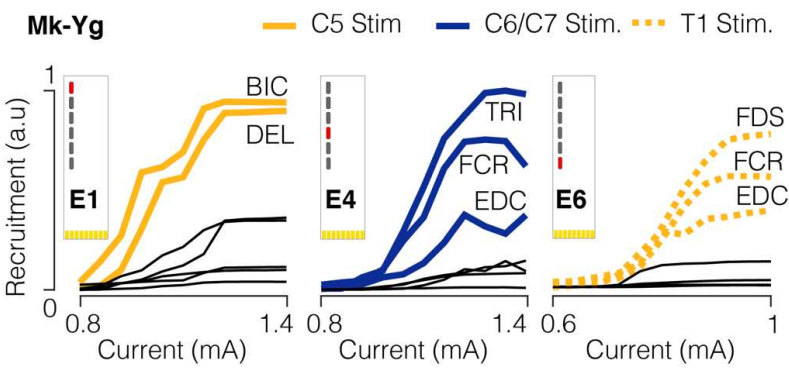

B Similar muscle recruitment in three monkeys

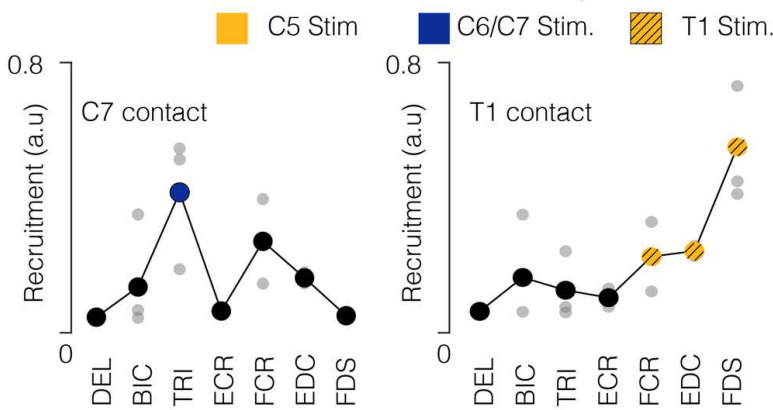

C Muscle recruitment during pulse trains
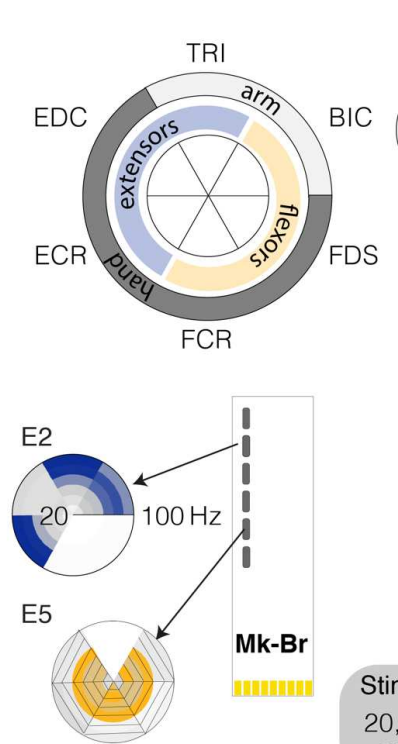

$\mathrm{Mk}-\mathrm{Br}$
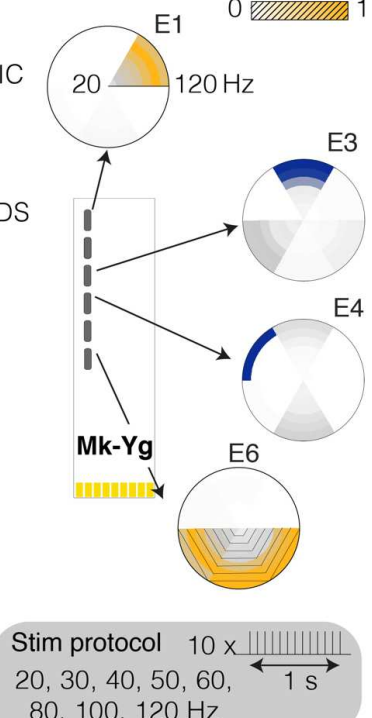

$80,100,120 \mathrm{~Hz}$

Figure 3. Muscle recruitment of spinal stimulation. (A) Examples of muscle recruitment obtained by stimulating $(1 \mathrm{~Hz})$ at $\mathrm{C} 5, \mathrm{C} 6 / \mathrm{C} 7$, and T1 spinal segments (Mk-Yg). (B) Average muscle activations elicited from $\mathrm{C} 7$ and $\mathrm{T} 1$ contacts in $\mathrm{n}=3$ monkeys (grey bullets: for each animal, average recruitment across all stimulation currents. Big bullets: mean of average recruitments across animals). (C) Muscle recruitment obtained during delivery of pulse trains in anesthetized monkeys. Recruitment was estimated by computing the energy of EMG signals for each muscle and each stimulation contact. Stimulation frequencies ranged from 20 to $120 \mathrm{~Hz}(n=2)$. For each muscle, energy values were normalized to the maximum value obtained across all frequencies and contacts. 
We next assessed the selectivity of the epidural interface. In propofol anaesthetized monkeys, we delivered asymmetric, charge-balanced biphasic pulses of EES at low repetition rate $(1 \mathrm{~Hz})$ at various current amplitudes from each contact. Minimum and maximum amplitude values were selected as the first subthreshold and first saturation current value respectively. As predicted ${ }^{53}$, different stimulation contacts generated muscle recruitment patterns that mirrored the segmental organization of cervical motoneurons (Figure 3A, Extended Data Figure 3A). Specifically, contacts located at C8/T1 level (caudal) elicited spinal reflexes mostly in the hand and forearm muscles, contacts located at $\mathrm{C} 7$ level elicited triceps and contacts located at C5/C6 recruited biceps and deltoids (rostral). Those results were consistent in all animals (Figure 3B). To ensure that this segmental selectivity translated into separate functional arm and hand movements, we delivered supra-threshold stimulation at various frequencies $(20-120 \mathrm{~Hz})$ from each contact in two animals (Mk-Br and $\mathrm{Mk}-\mathrm{Yg}$ ). Indeed, since recruitment of motoneuron is pre-synaptic, EES may not be able to produce sustained muscle activation because of frequency dependent suppression ${ }^{60}$. This effect is an observed substantial suppression of muscle evoked potentials during repetitive stimulation of the afferents. Instead, we observed large and sustained single joint movement during EES bursts. Muscle selectivity was preserved during long stimulation trains (Figure 3C) and different contacts elicited distinct joint movements (Video 1). When looking at the energy of the EMGs, we found a monotonic relationship between muscle activation and stimulation frequency in most of the upper arm muscles. However, not all muscles showed such clear frequency dependent responses (Extended Data Figure 3B). Moreover, peak-to-peak responses (Extended Data Figure 3C) were generally decreased during a burst at high

Arm movement is modulated by stimulation frequency
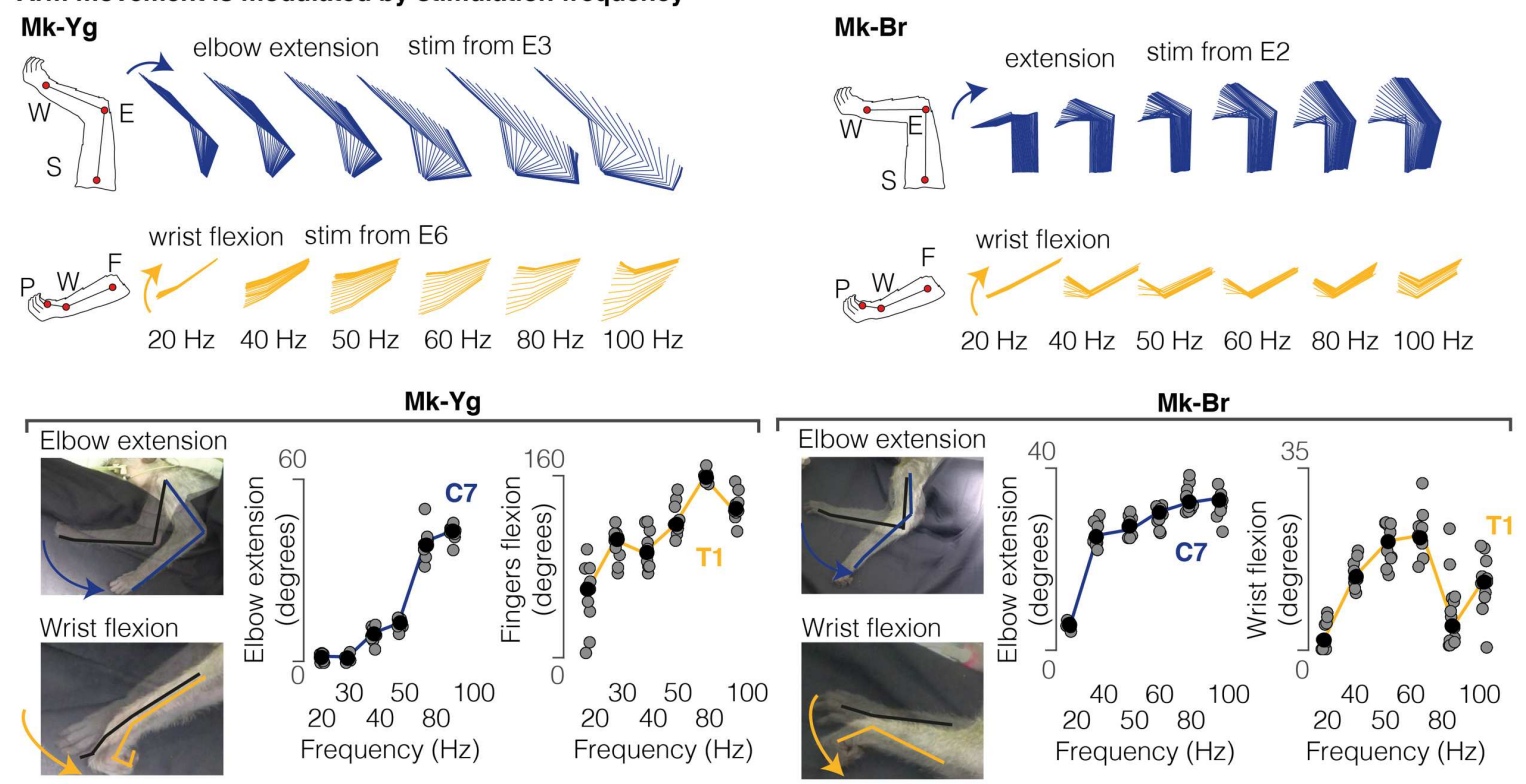

Figure 4. EES produces single joint movements in anesthetized animals. Top: stick diagram schematic of movements elicited by pulse-trains of stimulation in anesthetized conditions. Mk-Br: on the left, arm kinematic obtained by delivering stimulation at different frequencies from contacts number 2 and 5 (counting from the top); for $\mathrm{Mk}-\mathrm{Yg}$, on the right, arm kinematic obtained by delivering stimulation at different frequencies from contacts number 3 and 6 . Bottom: single joint angles excursions induced by stimulation at $\mathrm{C} 7$ (blue) and T1 (yellow) roots. Stimulation frequencies ranged from 20 to $100 \mathrm{~Hz}$ ( $\mathrm{n}$ $=2$ ). Black bullets: mean. Line: interpolation of the mean values. 
210 frequency but were not suppressed and tended to vary during the burst and while the movement 211 was produced. In summary, we found that single contacts of our spinal interface elicited 212 segmental recruitment of arm flexors, extensors and hand flexors. Bursts of stimulation from these 213 contacts produced sustained joint movements that were graded by stimulation frequency (Figure 214 4).

\section{Optimization of EES parameters}

We exploited these findings to determine the optimal contact location, stimulation amplitude and frequency that could sustain the production of movement phases of reach, grasp and pull that we observed in monkeys pre-injury. For example, contacts primarily targeting the $\mathrm{C} 7$ root (innervating triceps) produced clear elbow extension; instead, caudal contacts (C8/T1) elicited grasping and wrist movements (Figure 4A, Extended Data Figure 4). Kinematic output was modulated by

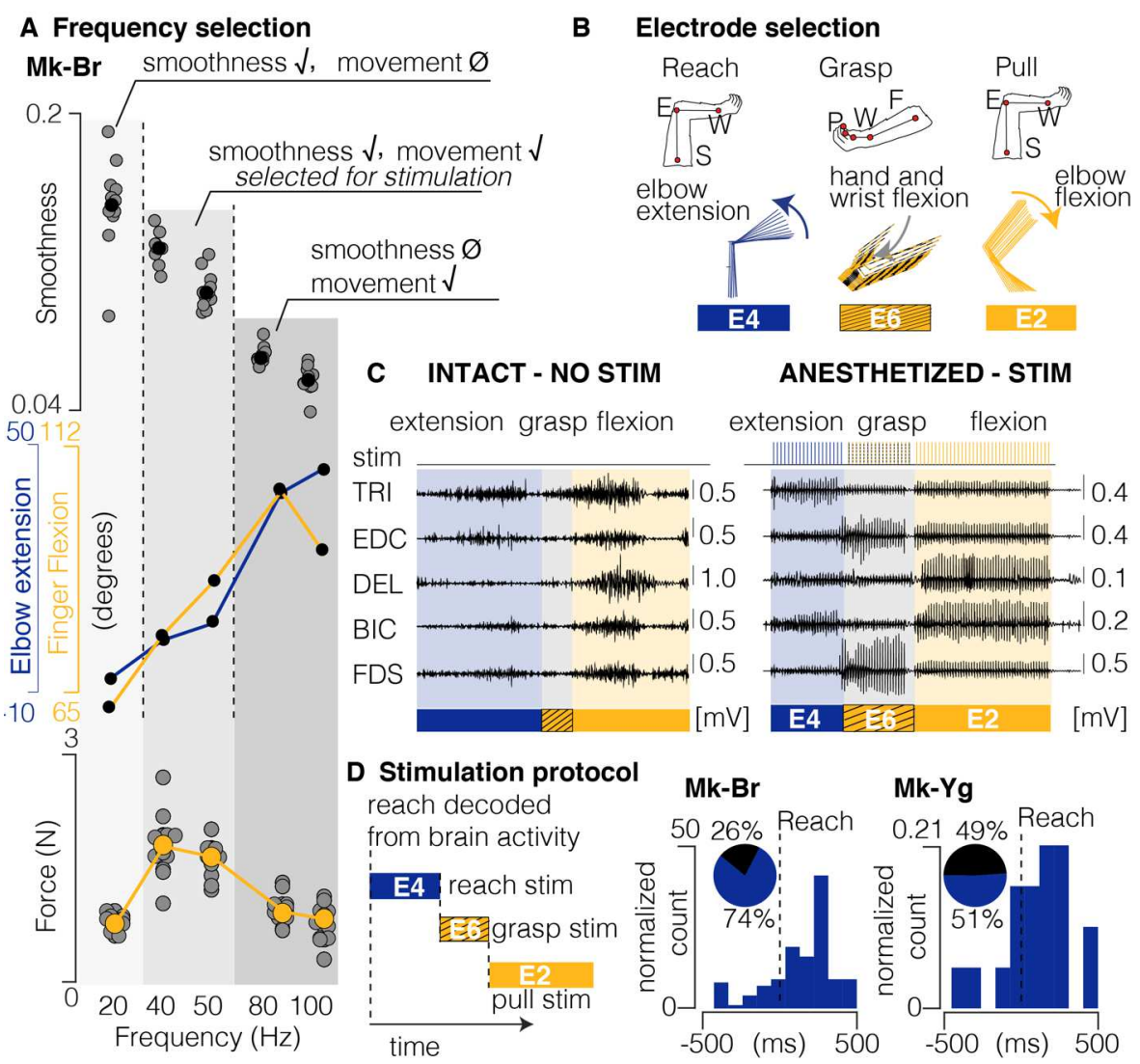

Figure 5. Design of stimulation protocol. (A) Combined representation of movement smoothness, elbow and finger flexion, and pulling force during anesthetized stimulation. Shades of gray highlight three frequency ranges that produce: (1) smooth trajectory, but little movement and low force $(2 \mathrm{~Hz})$, (2) smooth trajectory, extended movement and medium force (40 and $50 \mathrm{~Hz}),(3)$ abrupt and very extended movement and low force $(80$ and $100 \mathrm{~Hz})$. The range $40-50 \mathrm{~Hz}$ was selected as the best optimization of sufficient movement, smoothness and force production. (B) Schematic representation of arm and hand kinematics during stimulation delivered from the selection of three contacts to produce elbow extension (blue), hand and wrist flexion (yellow and black), and elbow flexion (yellow). (C) Example of comparison between EMG activity during intact movement (left) and movement elicited by chaining stimulation from the three selected contacts (right). (D) Scheme illustrating how stimulation is triggered from movement-related intra-cortical signals. On the right, online performances of movement attempt decoder in two animals with SCl. Pie charts represent percentage of predicted (blue) and unpredicted (black) reach events by our decoder. 
stimulation frequency (Figure 4B). By weighting joint excursion angles against movement smoothness ${ }^{61}$, we found that stimulation frequencies of $50-60 \mathrm{~Hz}$ (Figure 5A) produced smooth ${ }^{61}$ and full-range movements and maximal forces. Instead, movements elicited at frequencies lower than $40 \mathrm{~Hz}$ were often too weak to complete a joint movement and frequencies higher than $60 \mathrm{~Hz}$ produced either abrupt movements or incomplete movements (Figure 5A) probably because of the attenuation of muscle responses during repetitive stimulation of sensory afferents $53,60,62$ (Extended Data Figure 3C). Next, we identified three stimulation contacts that could consistently elicit arm extension (reach), hand flexion (grasp) and arm flexion (pull) (Figure 5B). By sequentially executing bursts on these three contacts, we could trigger whole arm movements that mimicked smooth ${ }^{61}$ and natural multi-joints movements (Figure 5C, Video 1). Specifically, extension, grasping and pulling movements produced clear EMG bursts as well as robust and smooth kinematics. These data demonstrate that with only three contacts, stimulation bursts can engage muscles that produce functionally relevant whole arm movements and sustained muscle activation and forces. Finally, we planned to link the delivery of these bursts to movement onsets information that we could extract from intra-cortical signals in real-time. We verified that also after $\mathrm{SCl}$, movement onsets could be reliably detected from intra-cortical signals (Figure 5D).

In summary, we optimized stimulation parameters to produce large and smooth single joint movements from single independent contacts and found that a frequency of 40 to $60 \mathrm{~Hz}$ was most effective. We then hypothesized that we could use bursts triggered at movement phase onset through these contacts to restore arm movements after SCl.

Cervical EES substantially improves arm and hand motor function after spinal cord injury We next tested whether our stimulation protocol could improve functional outcomes of upper limb movements after SCl. Specifically, we tested the efficacy of EES to improve muscle activation, pulling forces, functional task performance, and kinematic quality of three-dimensional movements after $\mathrm{SCl}$ when stimulation was on against stimulation off as a control. In all monkeys, the lesion led to substantial motor deficits of the left arm and hand.

While each monkey retained the ability to activate proximal shoulder and biceps muscles, elbow extension and hand functions were severely compromised. Severity of the impairment and extent of spontaneous recovery (Extended Data Figure 5) varied across monkeys because of the variability in lesion size (Figure 2E). Generally, animals showed severe paralysis immediately after lesion, and then gradually regained some movement capabilities (Extended Data Figure 5). Due to the initial impairment, immediately after the lesion, monkeys were not able to perform the behavioral task. Consequently, during the first week, we simplified the task by presenting an object close to the monkeys and triggering stimulation bursts manually to encourage the animal to perform the task. After the first week, all monkeys spontaneously attempted to perform the task, making it possible to link the delivery of movement-specific stimulation bursts to real-time detection of movement onset using intra-cortical signals. Whenever the monkeys strived for a reach, grasp or pull movement, we delivered bursts of stimulation promoting reach or grasp/pull respectively (movement specific EES). Outcomes were computed for each animal independently and compared between EES on and EES off. EES significantly enhanced muscles activity and forces (Figure 6B,D) compared to no stimulation. In terms of functional task performances, without stimulation, the monkeys were rarely capable of completing any part of the task (defined as reach, grasp and pull). Instead, with the support of EES, the rate of successes was significantly and robustly improved (Figure 6C, Video 2,3,4). Instead, when we used our interface to deliver continuous EES that was not related to movement onsets, only non-significant and modest improvements were observed in $\mathrm{Mk}-\mathrm{Br}$ while $\mathrm{Mk}-\mathrm{Yg}$ did not show ability to grasp and pull during continuous EES (Extended Data Figure 6A). Moreover, we analyzed trials in which stimulation bursts were not triggered at movement onset, for example when pull stimulation was erroneously 
triggered during reach. In these trials the reach movement was abruptly interrupted, and the animal did not complete the task (Extended Data Figure 6B, Video 5). In terms of movement quality, EES bursts triggered at movement onset significantly improved the overall quality of arm movements (Figure 6D). Indeed, principal component analysis (PCA) of

A

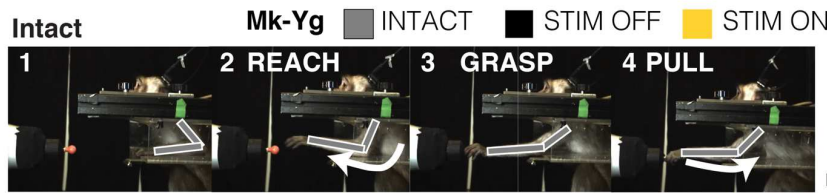

After SCl - stim OFF

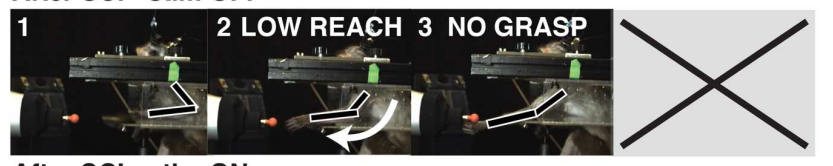

After SCI - stim ON

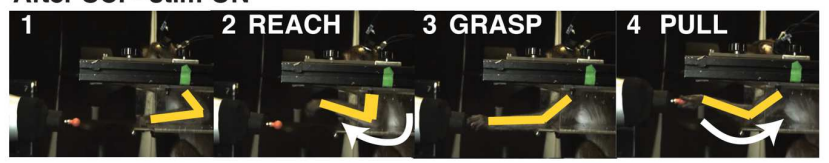

B EES improves muscle activation
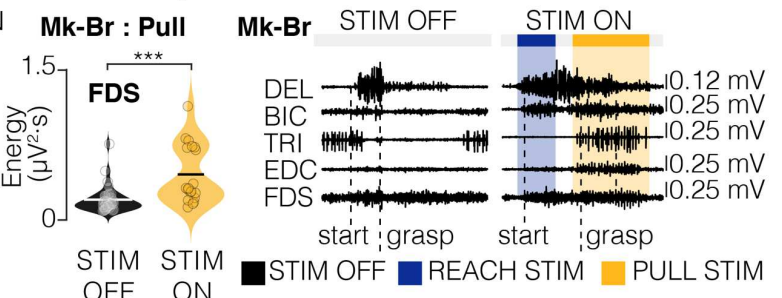

STIM OFF םREACH STIM PULL STIM

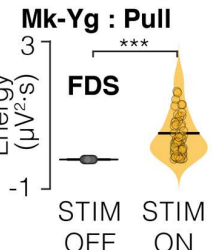

OFF ON
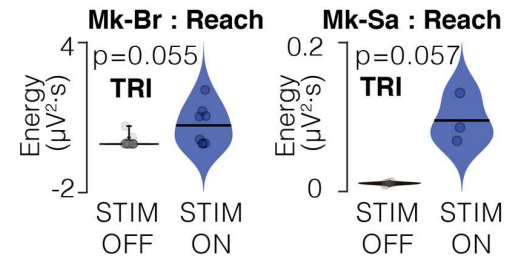

C EES improves task performance

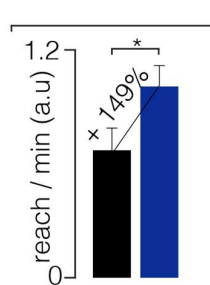
Mk-Br, week 3

D EES improves kinematics

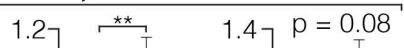
Mk-Yg

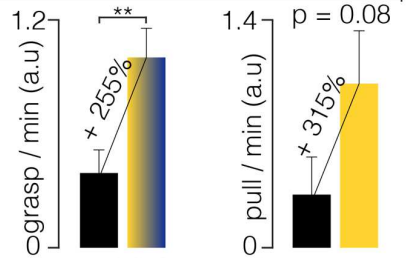

Mk-Sa, week 1 Mk-Yg, week 2
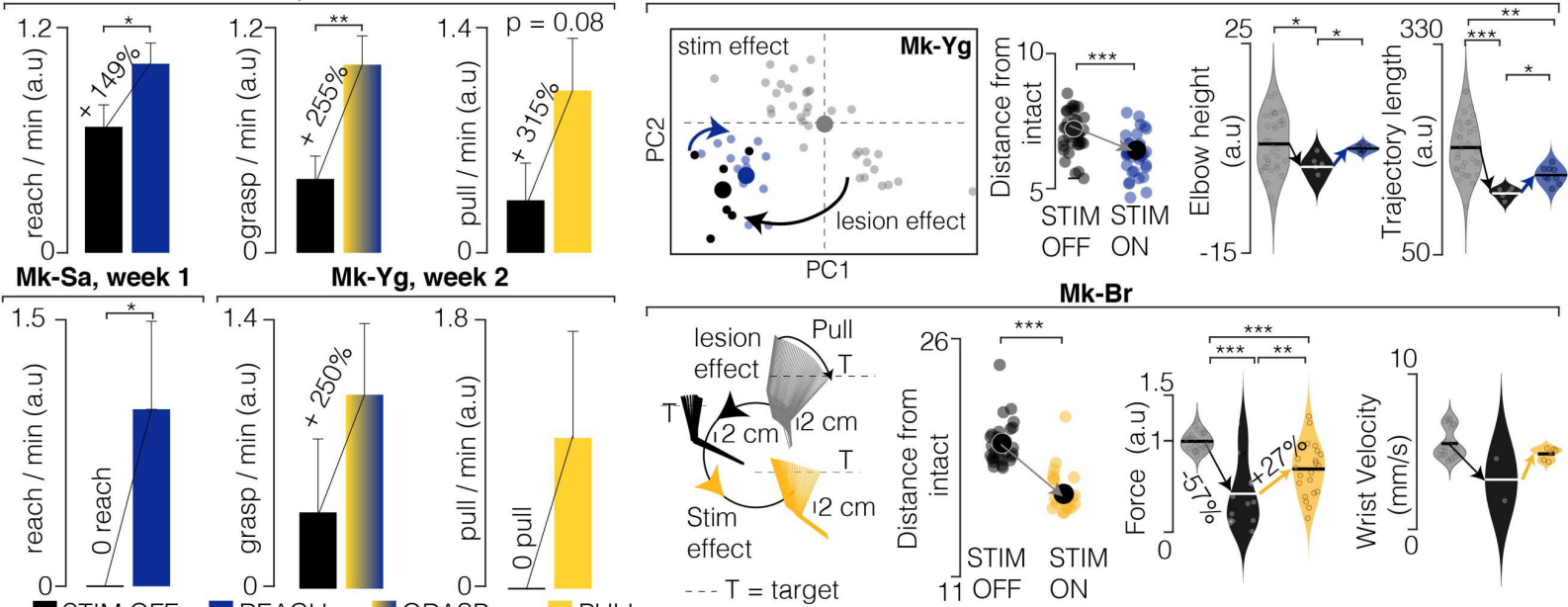

STIM OFF
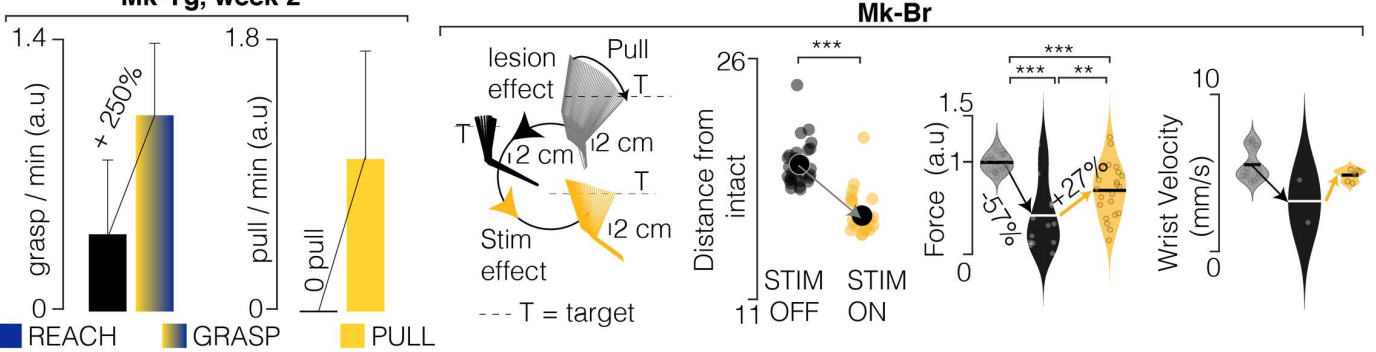

Figure 6. EES improves task performance, muscle strength and movement quality. (A) Snapshots of Mk-Yg performing the task before $\mathrm{SCl}$, after SCI without EES, and after SCI with EES. A full successful trial is composed of a reach, a grasp, and a pull. After $\mathrm{SCl}, \mathrm{Mk}-\mathrm{Yg}$ could only perform reaching movements without EES, while when EES was delivered the full task could be performed. (B) Violin plots of signal energy of triceps and FDS EMG profiles during reach (Mk-Br and $\mathrm{Mk}-\mathrm{Sa}$ ) and pull (Mk-Br and $\mathrm{Mk}-\mathrm{Yg}$ ). All individual data points are represented by bullets. Black lines correspond to the mean of the distribution. Statistical analysis with Wilcoxon Ranksum test. On the right, example raw EMG data after SCl with and without EES. (C) Bar plots report the rate of successful movements after SCl, without and with stimulation. Data are presented as mean \pm STD and normalized on the mean value in stimulation condition. Statistics was performed with Bootstrap. (D) Example PC analysis of kinematic features (See methods). Top-left, first and second PC space. Bottom left, stick diagram representation of arm kinematics during pull in intact conditions, after $\mathrm{SCl}$ without and with EES. At the immediate right (both bottom and top), euclidean distance in the feature space of trials without stimulation (black) and with stimulation (blue) from the centroid of the trials in intact condition. At the extreme right, example violin plots of movement quality features in the three conditions: intact, after $\mathrm{SCl}$, and after $\mathrm{SCl}$ with stimulation. Statistics with Wilcoxon Ranksum test. Asterisks: ${ }^{*} p<0.05,{ }^{* *} p<0.01,{ }^{* * *} p<0.001$. 
three-dimensional kinematic parameters (i.e., timing, force, arm trajectories, joint angles) revealed that during EES, movement kinematics were significantly closer to pre-lesion kinematics than the few successful movements performed without stimulation (distance from pre-lesion performances in the multi-parametric kinematic space, Figure 6D). Notably, animals sustained the weight of the arm and lifted their elbow more, performed wider movements, and generated stronger forces (Figure 6D), getting closer to normal kinematic trajectory patterns without any long-term training.

In summary, we showed that EES bursts triggered at movement phase onsets, improved muscle strength, task performance and quality of arm movements. This allowed monkeys to perform reach, grasp and pull movements that were otherwise not able to perform without EES.

285

286

287

288

289

290

291

292

293

294

295

296

297

298

299

300

301

302

303

304

305

306

307

308

309

310

311

312

313

314

315

316

317

318

319

320

321

322

323

\section{Sensory inputs can decrease EES-induced motor output}

We then investigated the role of spinal circuits and sensory inputs in the production of the movements that we observed. Indeed, since activation of motoneurons was pre-synaptic, spinal reflexes and sensory inputs can influence EES evoked spinal reflexes in the legs ${ }^{22,63}$. In order to exclude influences of residual supraspinal voluntary inputs, we conducted experiments under propofol anesthesia (Figure 7A). We then delivered bursts of EES targeting elbow flexion at varying stimulation frequencies in two distinct conditions (Figure 7B): in isometric and unconstrained conditions. In the isometric condition, we constrained the wrist, elbow and shoulder of the animal and measured force production at the wrist joint. Under unconstrained conditions we left the arm free to move under the effect of stimulation. This setup only differs from the sensory feedback generated at the load when pull forces are produced by EES. We found that EES induced EMG activity during unconstrained movement that was significantly different from the EMG activity induced during isometric movements (Figure 7B). In particular, overall EMGs and peak-to-peak amplitudes of elicited spinal reflexes were significantly lower when the arm was attached to a load (isometric) compared to when it was free to move. Albeit present at all frequencies, this difference was particularly important within the 40 to $60 \mathrm{~Hz}$ range, thus overlapping with the functional frequency ranged that we selected for our study.

These results show that force loads at the hand changed the input/output relationship between EES stimulation frequency and EMG activation so by decreasing muscle activity. Under anesthesia, only changes in sensory inputs can explain the observed changes on EES evoked muscle activity.

\section{Some residual cortical input is necessary for cervical EES to be effective}

The influence of spinal sensory inputs showed that EES output may be decreased because of spinal sensory inputs when loads are applied at the hand. This would decrease the efficacy of EES which is supposed to enhance force production. Therefore, to explain the results we obtained in behaving monkeys (Figure 6) we investigated the contribution of residual cortical inputs in the production of forces and movements during EES. Specifically, since cortical inputs actively modulate spinal circuits, they should be able to both enhance and suppress EES output by modulating spinal circuit excitability ${ }^{30}$. Since we showed that monkeys could use EES to amplify their movement and forces (Figure 6D) we focused on demonstrating that cortical inputs could also suppress unwanted EES-generated movements. We hypothesized that if monkeys did not want to move, EES would not produce the large joint movements that we observed when the monkeys were anesthetized. Therefore, we identified trials in which our decoder detected a falsepositive reach movement (Figure $\mathbf{7 C}$ ). In this situation our system would deliver a burst of stimulation even if the animal was not attempting to execute the task. We then compared intracortical activity from the primary motor cortex (M1) of $\mathrm{Mk}-\mathrm{Br}$ and $\mathrm{Mk}-\mathrm{Yg}$ during these falsepositive trials to the signals recorded during correctly detected trials. We identified trials where 


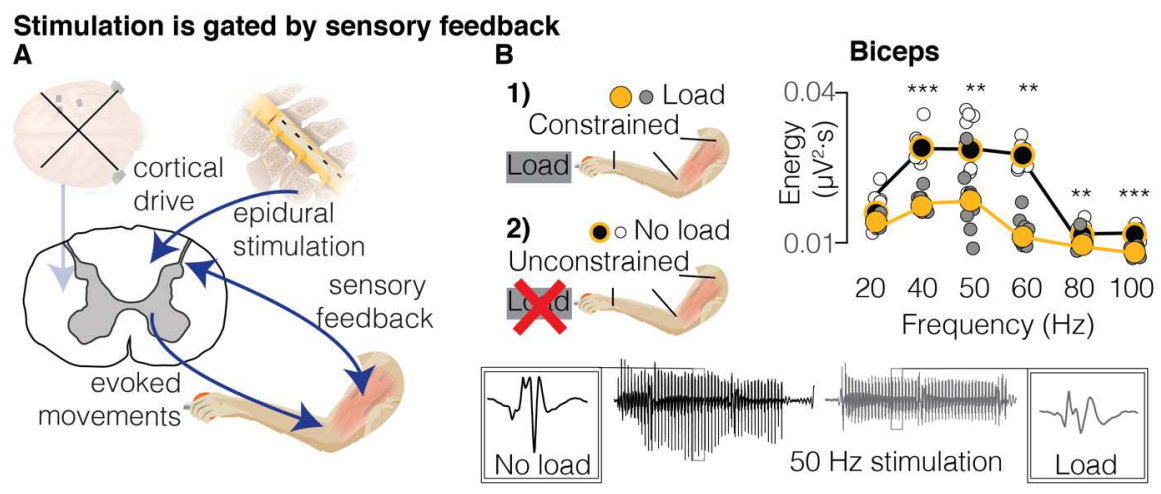

Stimulation is gated by cortical drive

C
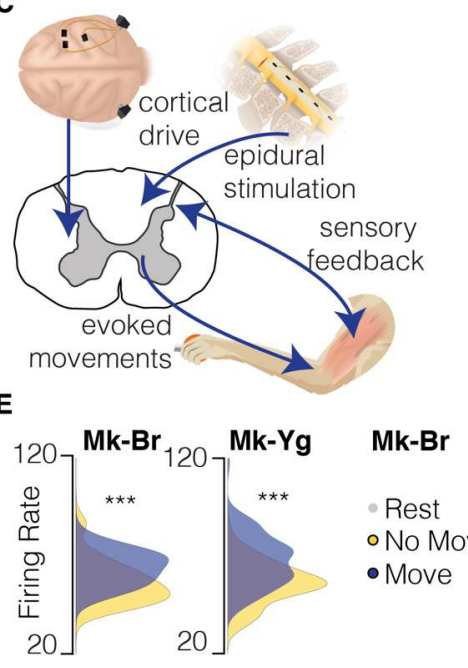

D EES without cortical drive NO MOVE

$\mathrm{Mk}-\mathrm{Br}$

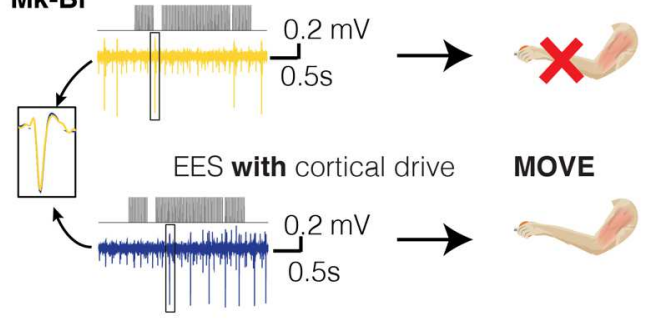

$\mathbf{F}$

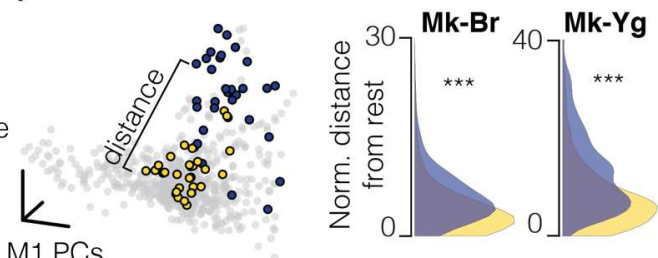

Figure 7. EES must be synchronized with motor intention. (A) Schematic of the interactions between EES and residual neural structures during anesthetized stimulation. During anesthesia, cortical control has no interaction, therefore EES interacts solely with sensory feedback spinal circuits. (B) Quantification of EMG activity during EES in two conditions: unconstrained arm (no load, black); arm constrained by load applied at the hand (load, gray). White and grey bullets: individual data points for no load and load conditions. Black and yellow bullets: mean values for no load and load conditions. Black and yellow lines: interpolation of mean values for no load and load conditions. On the bottom, example of EMG traces obtained during stimulation in the no-load (black) and load (gray) conditions. Stimulation artifacts have been removed. (C) Schematic of interactions between EES and residual neural structures during the performance of the behavioral task. EES interacts with descending cortical drive sent through residual pathways after $\mathrm{SCl}$, as well as with sensory spinal circuits. (D) Schematic illustrating the kinematic outcome of the interaction between EES and residual cortical inputs. The same EES pulse train (top) applied to $\mathrm{Mk}-\mathrm{Br}$ can result in different motor outputs: no movement output when the cortex is silent (yellow, top), movement is produced when the cortex is active (blue, bottom). (E) Distribution of average firing rates across all M1 channels during stimulation trains that evoked no movement (yellow) and movement (blue). (F) Left: State space view of M1 activity for all time points during rest (gray), successful stimulation (blue) and unsuccessful stimulation (yellow). The brain states during unsuccessful stimulation (yellow) overlapped with the rest states, while the successful stimulation (blue) did not. Right: we computed a relative Mahalanobis distance between the two stimulation conditions and the cluster of neural states at rest. For both monkeys, neural states during stimulation periods with no movement were close to rest. 
found that the overall firing rates of all units in motor cortex was significantly higher when EES produced movement (Figure 7E) than when it did not. This suggested that movement happened only if the motor cortex was active, despite EES was delivered at amplitudes that generated large joint movements when the same monkey was anesthetized. To further validate this hypothesis we applied dimensionality reduction using Principal Component Analysis to the firing rates in each electrode and reduced the M1 population activity to low-dimensional states ${ }^{64}$. In this lowdimensional space each point represents the global neural state of the motor cortex at a given time point (Figure 7F). We compared the neural states present when EES was associated movements and those when EES was not associated movement with the neural states associated to rest, e.g. when the monkeys were resting before the go signals between trial repetitions. When looking at the spatial distribution of neural states, trials in which EES was not associated to movement seemed to overlap with states of rest. We then computed the distance between each neural state to the subspace representing neural states at rest and found that the neural states associated to movements during EES were significantly further away from neural states at rest than neural states associated to EES and no movement. In summary, we found that the motor cortex activity was similar to the activity at rest whenever we delivered EES but the monkey did not move (Figure 7F). Instead, the monkey moved when the motor cortex was significantly active. This implies that the residual cortical inputs via direct and indirect pathway can either suppress or enable movement during EES.

\section{Discussion}

We showed that EES of cervical spinal cord immediately enhanced muscle activation and strength, task performances and movement quality during a natural-like reach and grasp task in monkeys with unilateral cervical SCI compared to no stimulation controls in three monkeys. Importantly, our technique allowed monkeys to support the weight of their arm during reach, grasp and pull movements. These results are important in light of clinical translation of our technology. Stronger forces and better arm weight bearing can empower patients with the capacity to perform a larger spectrum of movements than they would normally be capable of doing without the need of support. This may provide for more independence in daily living as well as better outcomes of physical therapy.

\section{Exploiting subject-specific anatomy to simplify technology}

We obtained our results with relatively simple stimulation protocols that engaged up to three monopolar contacts (one for reach, one for grasp and one for pull). The combination of simple bursts through these contacts enabled whole arm multi-joint movements. We believe that the design of our interface was key to achieve this result. The dorsal roots are a robust anatomical target that we could easily identify through standard imaging to personalize surgical planning and interface design. A similar surgical planning approach can be imagined in humans where MRIs and CT can guide surgical planning ${ }^{51,65}$.

Our results were enabled by the relative mapping between each dorsal root and the rostro-caudal distribution of motoneurons in the cervical spinal cord, which is similar in monkeys and humans ${ }^{53,55,66}$. The anatomical separation of roots in the cervical enlargement allowed us to recruit each root independently which generated distinct joint movements to a degree that was not observed in applications of EES for the lower limbs ${ }^{49}$. Stimulation of the C6 root elicited distinct arm flexion, C7 stimulation produced arm extension and C8/T1 stimulation produced hand grasp. However, similarly to other spinal cord stimulation studies we could not identify contacts that selectively produced finger extension $18,67,68$. This is likely caused by the overlap of extensor motorpools in the forearm ${ }^{55,66}$ but possibly also because flexors may be biomechanically stronger and 
dominate hand kinematics in the case of co-contraction at rest. Despite these limitations in specificity, we were able to restore a whole three-dimensional arm movement by solely detecting movement onset signals to trigger pre-determined stimulation bursts through two or three contacts. Unlike FES, this is possible because EES activates cervical motoneurons via pre-synaptic inputs thus allowing modulation of elicited muscle responses that can compensate for reduced specificity ${ }^{30,49}$.

\section{Supporting arm movement phases independently}

Differently from previous pilot applications of spinal cord stimulation of the cervical spinal cord ${ }^{35,36}$, we utilized a selective interface to independently support each movement phase rather than providing continuous stimulation to the whole spinal cord. This approach was shown to be more effective in animal models and humans than continuous stimulation in the sense that it was able to immediately produce coordinated locomotion compared to continuous stimulation that instead required long training periods $28,48,49,49,56$. In the case of the upper limb we believe that this approach was critical. Indeed, while continuous stimulation did provide some level of facilitation, it failed to entirely promote grasp and pull in one of the monkeys. Perhaps the intrinsically unstructured nature of arm and hand control makes a continuous stimulation approach less effective than it is in locomotion that instead has an intrinsic repetitive structure ${ }^{38}$. For example, stimulation parameters that promote grasp, may impair reach if they are delivered continuously throughout movement. Indeed, when a pull stimulation was triggered at mid-reach it generated the interruption of the reach movement. Perhaps a different interface design or lower stimulation amplitudes could be used to optimize continuous stimulation protocols, but it would be at the expense of power of elicited movements potentially preventing the weight bearing component necessary for three-dimensional movements. In summary, the complex articulation of arm and hand movements may exacerbate the difference in efficacy between continuous and phasespecific stimulation protocols that was already observed for EES in locomotion, possibly explaining the difference in effect size that was obtained so far for application in the upper limb.

\section{The role of sensory feedback and residual cortical inputs in cervical EES}

We showed that sensory feedback when the hand was constrained to a force load reduced the EMG power produced by EES compared to free movements. This is likely caused by afferent inhibitory feedback coming from lb afferents. Unfortunately, lower muscle power while resisting a force load would decrease the clinical usability of this technology. We believe that this phenomenon is particularly relevant for the upper limb. Indeed, also during EES of the lumbosacral cord, the EES motor output is influenced by sensory inputs 22,63 , however sensory inputs are instrumental for locomotion and heavily contribute to the generation of the repetitive movement patterns that are required to walk ${ }^{16,22,23,38,69}$. Therefore, in the case of locomotion these inputs amplified and sustained EES-induced activity ${ }^{16,22,23,28}$. Instead arm and hand movements are produced by an unstructured sequence of primitive movements ${ }^{41}$ and reflexes ${ }^{45}$ in parallel with a sophisticated gating of sensory inputs through mechanisms such as pre-synaptic inhibition ${ }^{8,70}$. Therefore, residual cortical inputs become instrumental to obtain arm and hand movement with EES as shown by our analysis of intra-cortical signals during the production of movement of EES. Our lesions were non-complete and while most of the cortico-spinal tract was transected, multiple residual descending pathways were spared. These indirect inputs could have been used by the animals to mediate the inputs required to integrate EES and sensory inputs to produce voluntary movements. In summary, we believe that even during phase-specific EES residual cortical inputs play a critical role in enabling arm movement for cervical EES.

\section{Clinical significance}


The most important challenge for clinical translation of EES to humans concerns the role of residual inputs. Our data show that some level of residual inputs is likely required to enable movement. However, previous studies showed that even completely paralyzed subjects retain residual but functionally silent descending inputs $25,32,51$. Therefore, while overall efficacy may modulate with injury severity, even severely injured patients may obtain benefits from cervical EES. Concerning complexity of our system, in our study we detected movement onsets from intracortical activity which may be seen as a limitation for a realistic implementation of our protocol in clinical settings. However, given the simplicity of our protocol which is essentially constituted by alternation of pre-defined bursts, brain recordings may not be required in clinics. Indeed, most patients suffer from a severe but incomplete paralysis ${ }^{51,71}$, which spares some residual muscle activity in few muscles. While this residual activity is not sufficient to produce functional movements, it can be reliably detected and used to trigger stimulation bursts with standard clinical technologies ${ }^{49,51}$. In summary, we believe that by exploiting the functionality of residual spinal circuits and supra-spinal inputs, cervical EES constitutes a simple yet robust approach to the restoration of arm motor control with significant translational potential.

\section{Acknowledgements}

The authors would like to thank Jacques Maillard and Laurent Bossy for the care provided to the animals, Dr Eric Schmidlin and Dr Simon Borgognon for their help with anaesthesia and surgery preparations, Dr Marion Badi for her help and advice during experiment preparations and experimental procedures, Dr. Andrina Zbinden for her contribution to the health survey of the monkeys, André Gaillard and Andrea Francovich for their help with the implementation of the hardware and the students of the University of Fribourg Amélie Jeanneret, Alen Jelusic, Laora Marie Jacquemet and Samia Borra for their help in processing data.

\section{Funding}

The authors would like to acknowledge the financial support from the Wyss Center grant (WCP 008) to MC, GC and TM, an industrial grant from GTX medicals to GC and MC; the Bertarelli Foundation (Catalyst Fund Grant to MC and TM and funds to SL) a Swiss National Science Foundation Ambizione Fellowship (No. 167912 to MC), The European Union's Horizon 2020 research and innovation program under the Marie Skłodowska-Curie grant agreement no. 665667 (GS) the Swiss National foundation grant BSCGI0_157800 (SL), a Whitaker International Scholars Program fellowship to MGP, and an internal pilot grant of the University of Fribourg to MC.

\section{Author Contributions}

MC, BB and SC conceived the study; BB, MGP, and TM designed and implemented the hardware and software tools; SC designed the behavioral task and training strategy; GS and SL designed and manufactured the implantable interface; BB, SC, MGP and MC conducted the experiments; $\mathrm{BB}, \mathrm{SC}, \mathrm{MGP}$ and KZ performed the data analysis; SC, MD and MK trained the animals; SC, KG, NJ and QB processed the histological data; JB, GC and MC designed surgical implantation strategies and stimulation strategies. GC and JB, performed surgical implantations and lesions. EMR and MC implemented and supervised procedures on monkeys; MC, BB, SC and MGP wrote the manuscript; all authors edited the manuscript; SL, TM, JB, GC and MC secured funding for the study; MC supervised the study.

\section{Competing Interests}


471 G.C., J.B., S.L., M.C., B.B. and K.Z. hold various patents in relation to the present work. G.C., 472 S.L. and J.B. are founders and shareholders of GTX medical, a company developing an EES473 based therapy to restore movement after spinal cord injury.

474

475

476

Data and materials availability

477

All software and data will be available upon reasonable request to the corresponding author.

478

479

480

481 

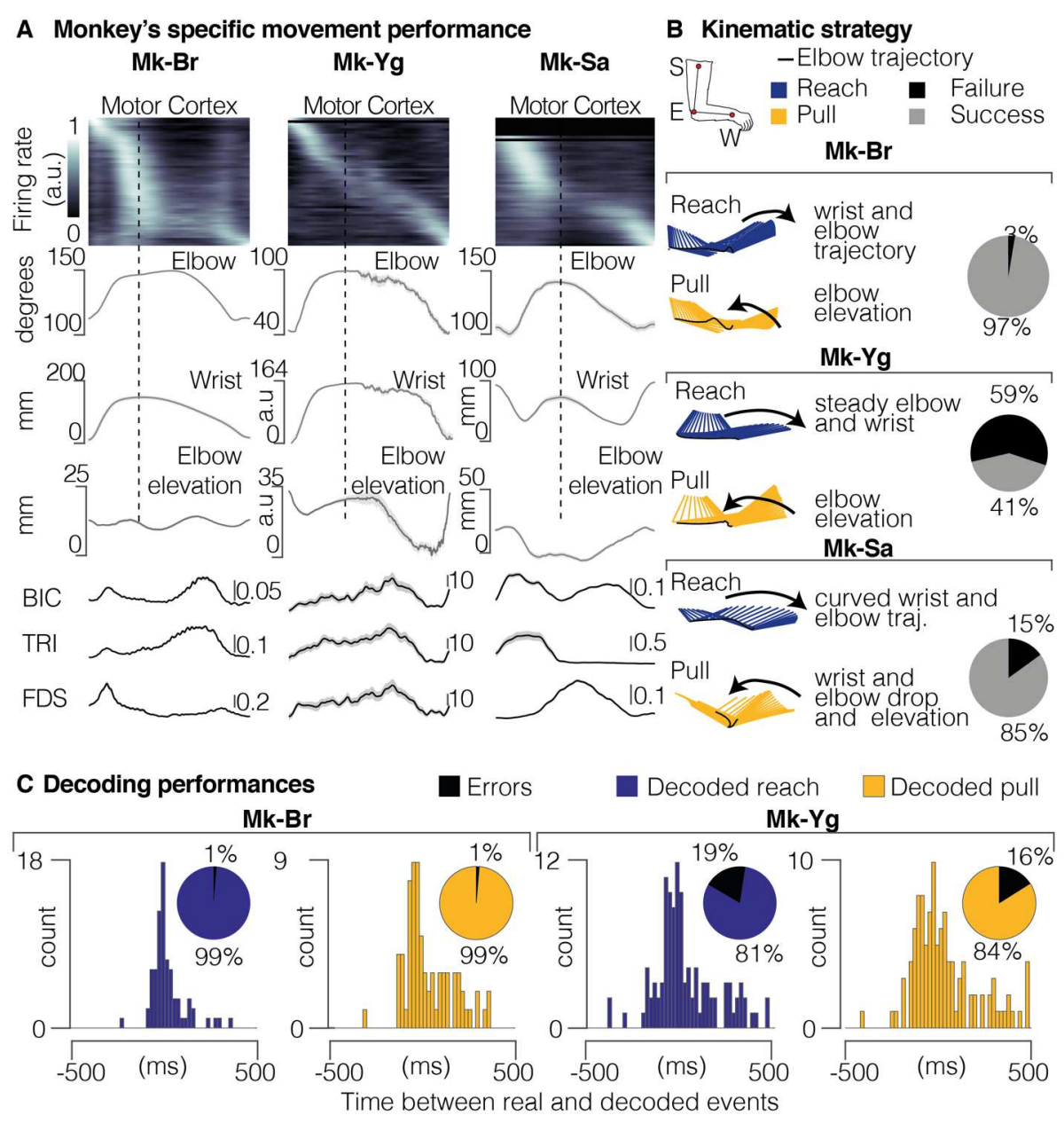

Extended Data Figure 1. (A) Portfolio of signals recorded during intact movement for each animal. These signals have been recorded during the experimental session prior to the lesion. Black line corresponds to the mean profile across all trials, shaded area shows the SEM across all trials. (B) Kinematic strategies implemented by each monkey. Stick diagrams representations of the arm kinematic during reach (blue) and pull (yellow). The black line highlights the elbow trajectory. Pie charts represent the percentage of success and failure in task performance before lesion. (C) Offline decoding performance for Mk$\mathrm{Br}$ and $\mathrm{Mk}-\mathrm{Yg}$ before lesion. Histograms show the timing accuracy of detected reach (blue) and grasp (yellow) events. Pie charts (inset) show the percentage of correctly identified events. 
A

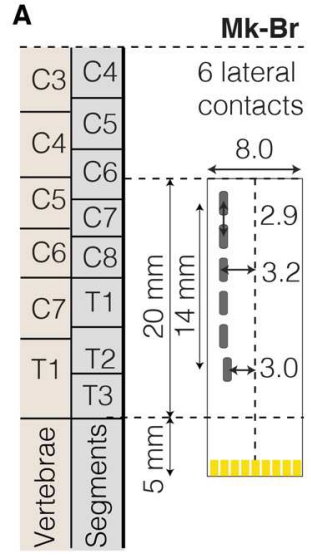

Mk-Yg

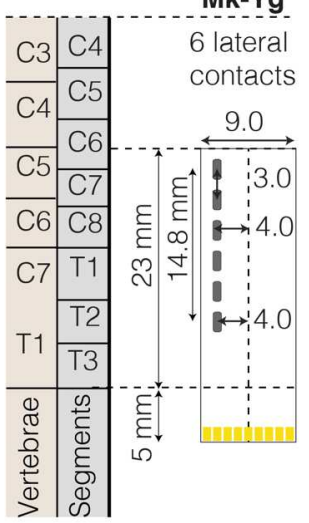

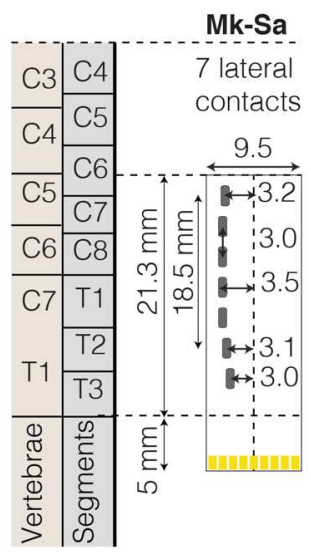

B Position stability of the implant over time C
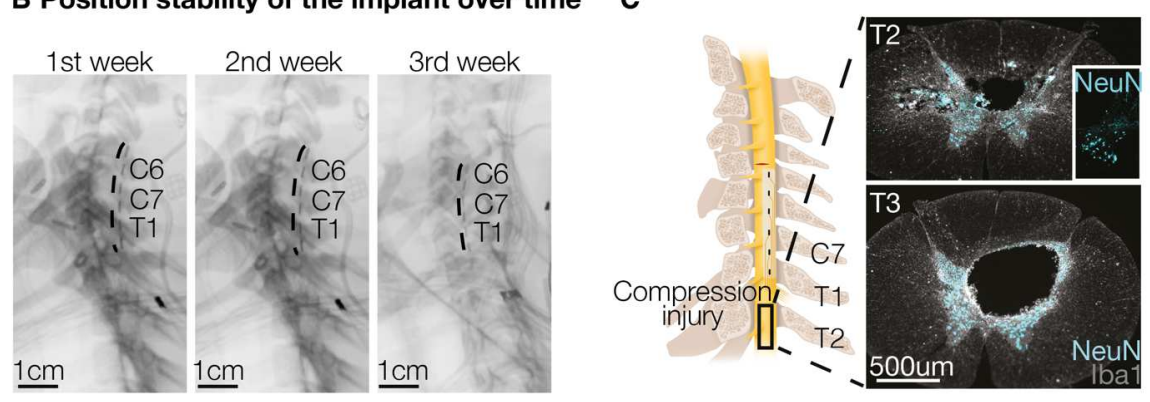

Extended Data Figure 2. (A) Personalized design of the epidural implant for each animal. All measures are in millimeters. Yellow traces at the bottom of the electrode identify connectors. (B) Position stability of the epidural array over time, illustrated through Xrays imaging taken during 3 consecutive weeks after the implantation. (C) Compression injury at the insertion level of the array (T2-T3 segment) in Mk-Br, discovered postmortem, stained with NeuN (neuronal cell bodies) and Iba1 (microglia). 


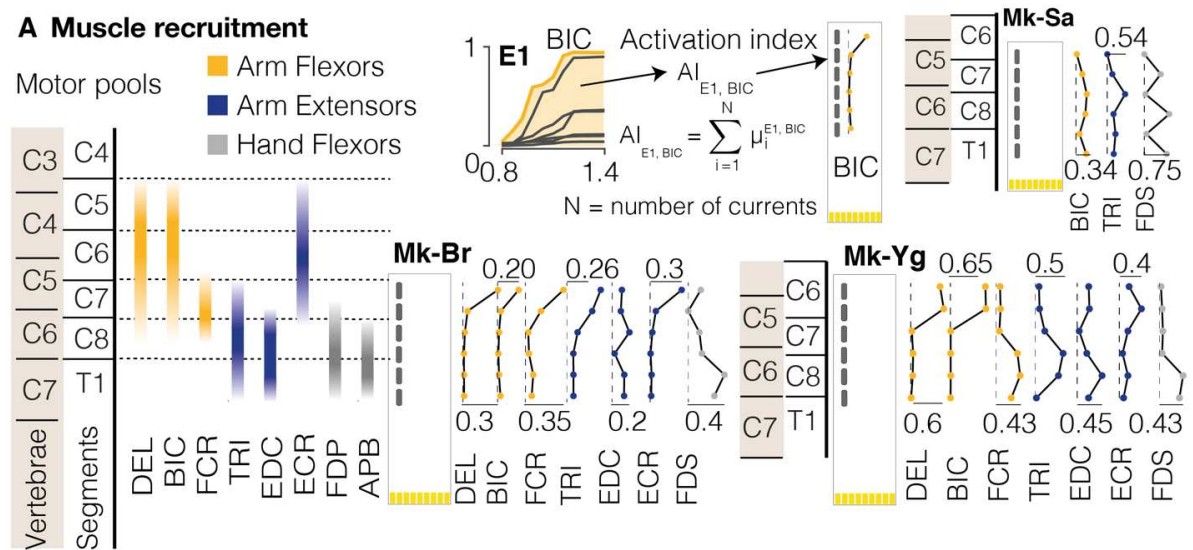

B Graded muscle activation during train pulses
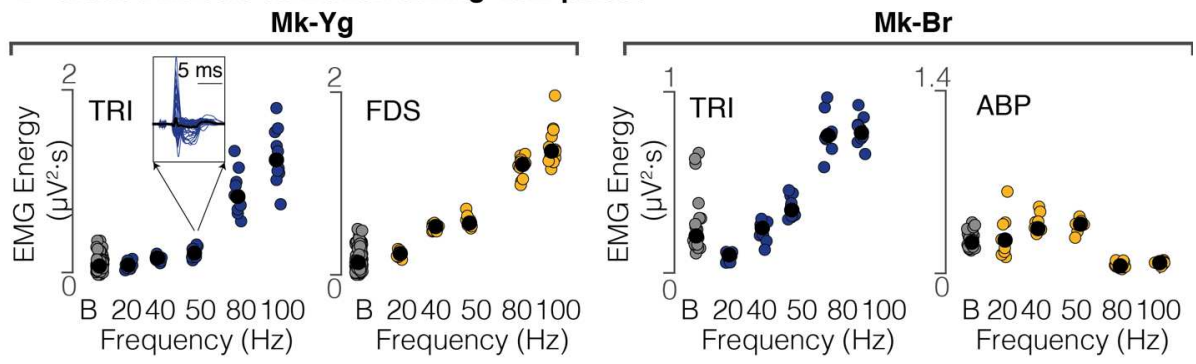

C Muscle responses are modulated at higher frequencies

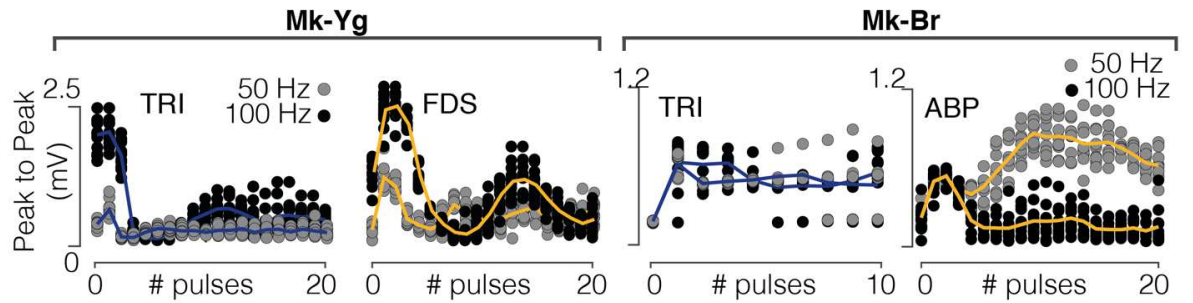

Extended Data Figure 3. (A) Single pulse muscle recruitment for each animal, contact, and muscle. Bullets identify the Activation Index (computation illustrated in the schematic above). Each bullet corresponds to a specific muscle (on the $x$-axis) and a specific contact (on the y-axis, illustrated in the implant schematic on the left). Lines connect bullets corresponding to the same muscle, across different stimulation contacts. (B) Energy of EMG signals of triceps (Mk-Br and Mk-Yg), Flexor Digitorium Superficialis (Mk-Yg) and abductor pollicis (Mk-Br) muscles, following pulse-train stimulation at different frequencies (on the $\mathrm{X}$-axis). Black bullets represent mean values. (C) Evolution over time of the peak to peak value of stimulation evoked responses during a stimulation burst. Each plot shows the evolution for a specific muscle following pulse-train stimulation at 50 and $100 \mathrm{~Hz}$. Triceps is shown for Mk-Br and Mk-Yg, Flexor Digitorium Superficialis for Mk$\mathrm{Yg}$ and abductor pollicis for $\mathrm{Mk}-\mathrm{Br}$. Each data point is represented as a bullet and lines represent mean values over time. 
A Arm movement is modulated by stimulation frequency
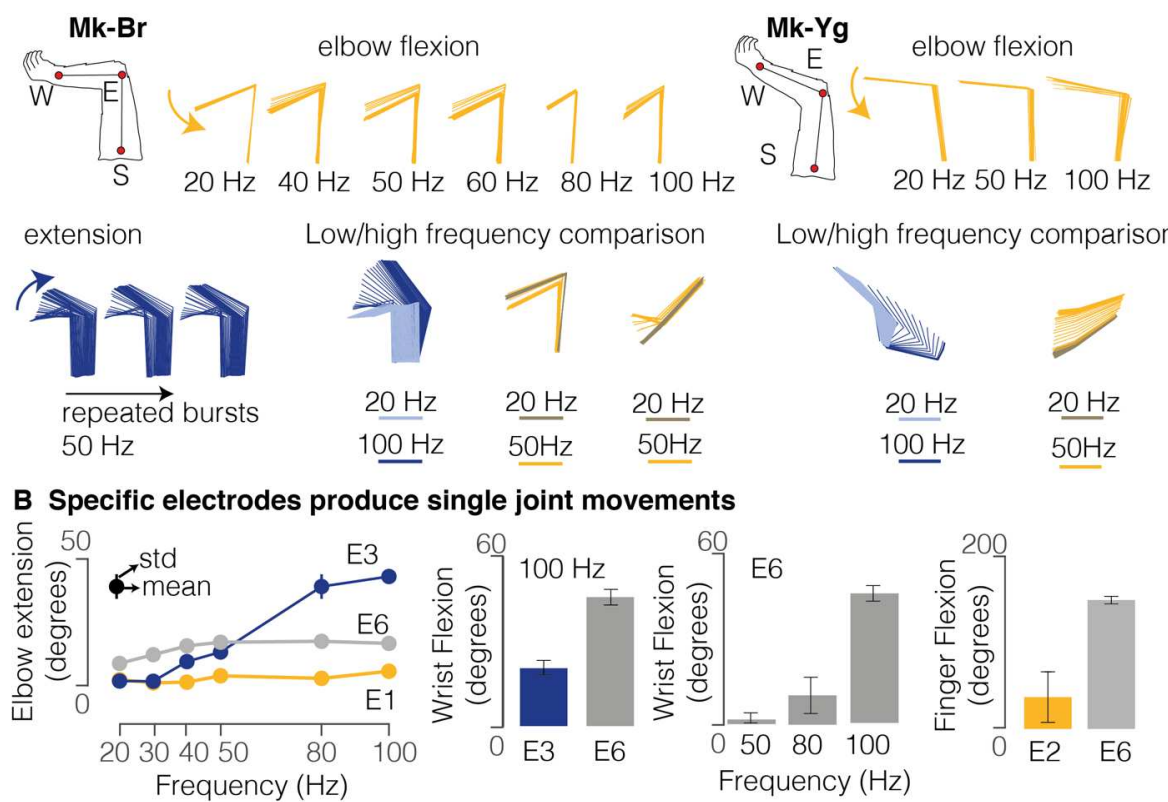

Extended Data Figure 4. (A) Stick diagram schematic of movements elicited by pulsetrains of stimulation in anesthetized conditions. Mk-Br: on the left, arm kinematic obtained by delivering stimulation at different frequencies from contact number 5 , on the bottomleft, arm kinematics obtained by repetitive delivery of a burst at $50 \mathrm{~Hz}$; on the bottom right, superimposition of stick diagrams obtained with stimulation at $20 \mathrm{~Hz}$ and at higher frequencies $(50$ or $100 \mathrm{~Hz}$ ) from different contacts. For Mk-Yg: arm kinematic obtained by delivering stimulation at different frequencies from contact number 2 and superimposition of stick diagrams obtained with stimulation at $20 \mathrm{~Hz}$ and at higher frequencies (50 or $100 \mathrm{~Hz}$ ) from different contacts. (B) On the left, elbow extension produced by stimulation at different frequencies. Bullets represent the mean value across different pulse-trains, and lines represent the standard deviation. Note that most of times standard deviation is so small that it remains hidden from the bullet. At the immediate right, wrist flexion obtained by stimulation through different contacts (at $100 \mathrm{~Hz}$ ) and at different frequencies (from contact number 6). At the extreme right, wrist flexion obtained by stimulation through different contacts. Values are plotted as the mean \pm STD. 


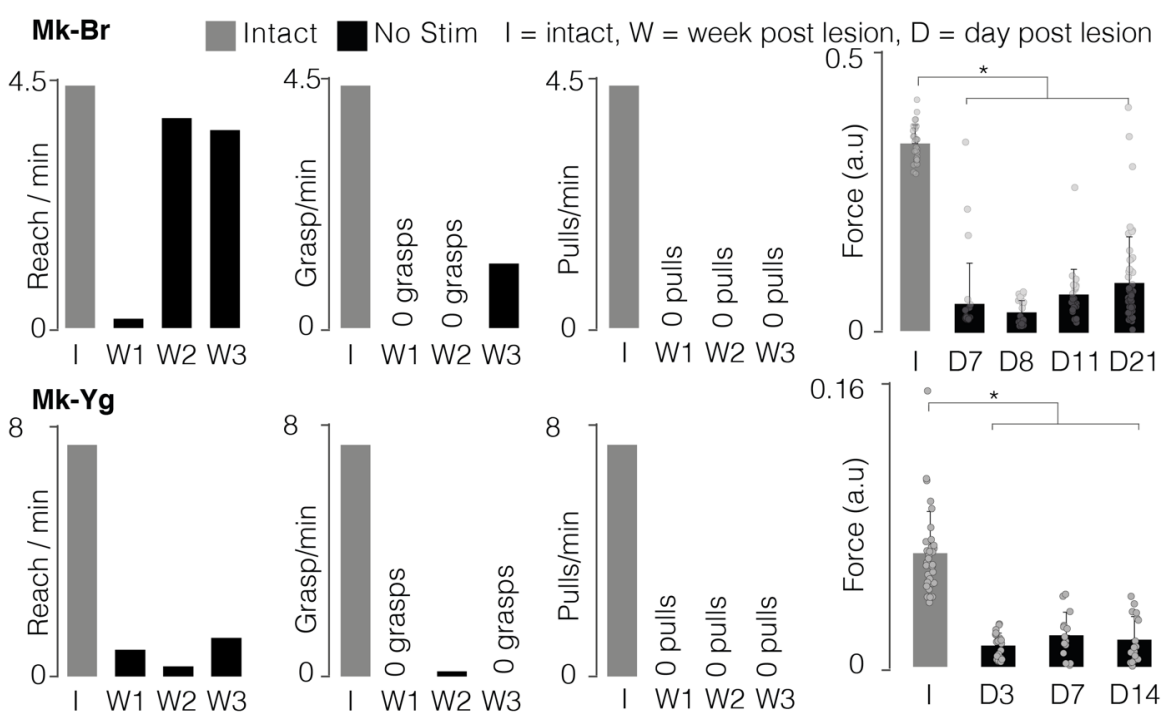

Extended Data Figure 5. Left: For $\mathrm{Mk}-\mathrm{Br}$ and $\mathrm{Mk}-\mathrm{Yg}$, evolution (in weeks) of rates at which reach, grasp or pull movements are performed after SCI (black), compared to the performances before injury (gray). Right: Evolution (in days) of pull force after SCl without stimulation. Values are plotted as the mean \pm SEM. Statistical analysis was carried out with Wilcoxon Ranksum test. 


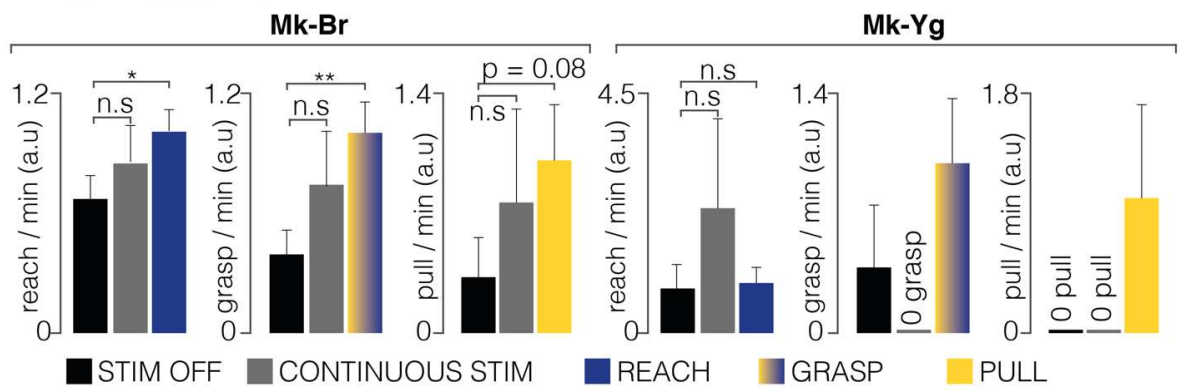

B Effect of stimulation timing

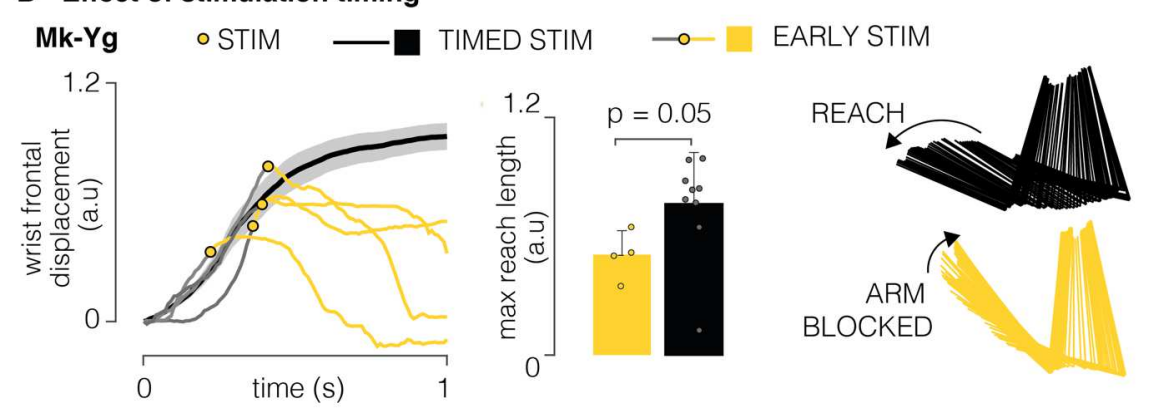

Extended Data Figure 6. (A) Bar plots report the rate of successful movements after $\mathrm{SCl}$, without stimulation (black), with continuous stimulation (gray) and with phasedependent stimulation (blue or yellow) for $\mathrm{Mk}-\mathrm{Br}$ and $\mathrm{Mk}-\mathrm{Yg}$. Data are presented as mean \pm STD and normalized on the mean value in stimulation condition. Statistics was performed with Bootstrap. (B) Left: wrist frontal displacement in trials in which pull stimulation was erroneously triggered during reach (gray and yellow), compared to trials in which pull stimulation was not delivered (black). Yellow bullets highlight the instant at which stimulation was delivered: yellow lines highlight the trajectories during and after stimulation. Middle: barplot of the length of the reach movement when pull stimulation was erroneously delivered and when pull stimulation was not delivered. Data are presented as mean \pm STD. Right: stick diagram of arm kinematics during reach without (black) and with (yellow) erroneous pull stimulation. 


\section{Materials and Methods}

\section{Animals involved in the study}

All procedures were carried out in accordance to the Guide for Care and Use of Laboratory Animals ${ }^{72}$ and the principle of the 3Rs. Protocols were approved by local veterinary authorities of the Canton of Fribourg (veterinary authorization No 2017_04_FR and 2017_04E_FR), including the ethical assessment by the local (cantonal) Survey Committee on Animal Experimentation and final acceptance by the Federal Veterinary Office (BVET, Bern, Switzerland). Three adult female Macaca Fascicularis monkeys were involved in the study (Mk-Sa 9 years old, $4.0 \mathrm{~kg}, \mathrm{Mk}-\mathrm{Br} 3$ years old, $3.4 \mathrm{~kg}, \mathrm{Mk}-\mathrm{Yg} 3$ years old, $4.0 \mathrm{~kg}$ ). Animals were not food deprived, could freely access water at any time and were housed in collective rooms designed in accordance to the Swiss guidelines (detention in groups of 2-5 animals in a room of at least $45 \mathrm{~m}^{3}$ ). Rooms were enriched with toys, food puzzles, tree branches and devices to climb and hide, as well as access to an outdoor space of 10-12 $\mathrm{m}^{3}$ (see www.unifr.ch/spccr/about/housing). Detailed information on which animals were involved in specific experimental procedures are reported in Supplementary Table 1.

\section{Surgical procedures}

513 For each animal, we performed three surgical procedures, (1) intracortical electrodes implantation, 514 (2) intramuscular electrodes implantation, and (3) epidural implant insertion and spinal cord injury. Mk-Sa deviated from this protocol. Mk-Sa was first implanted with the epidural interface before injury, however an infection occurred and resulted in the explanation of the lead to treat the infection. After recovery, the animal was re-implanted, and lesion performed following the same protocol of $\mathrm{Mk}-\mathrm{Br}$ and $\mathrm{Mk}-\mathrm{Yg}$. All the surgical procedures were performed under full anaesthesia induced with midazolam (0.1 mg/kg, i.m.), methadone $(0.2 \mathrm{mg} / \mathrm{kg}$, i.m.), and ketamine $(10 \mathrm{mg} / \mathrm{kg}$, i.m.) and maintained under continuous intravenous infusion of propofol $(5 \mathrm{ml} / \mathrm{kg} / \mathrm{h})$ and fentanyl (0.2-1.7 ml/kg/h) using standard aseptic techniques. A certified neurosurgeon (Dr. Jocelyne Bloch, CHUV, Lausanne, Switzerland) performed all the surgical procedures.

During the first surgical procedure, we implanted multi-microelectrode arrays in the primary motor cortex (M1-42 channels), ventral premotor cortex (PMv-32 channels) and primary somatosensory cortex (S1-42 channels) for a total of 128 channels for Mk-Br and Mk-Yg (Blackrock Microsystems, $400 \mu \mathrm{m}$ pitch and electrodes tip lengths $1.5 \mathrm{~mm} 1.5 \mathrm{~mm}$ and $1 \mathrm{~mm}$ for M1, PMv and S1 respectively). Instead, Mk-Sa was implanted with 2 microelectrode arrays of 64 channels each and pitch of 1.5 and $1 \mathrm{~mm}$ in M1 and PMd respectively. Functional motor areas of the arm were identified through anatomical landmarks and intra-surgical micro-stimulation. In order to access the brain areas of interest we performed a $20 \mathrm{~mm}$ diameter craniotomy and we incised the dura. The arrays implantation was achieved using a pneumatic compressor system (Impactor System, Blackrock Microsystems). A pedestal (Pedestal A) was then fixated to a compliant titanium mesh (Medtronic Ti-Mesh) modelled to fit the skull shape and implanted in a previous surgery a few 
weeks earlier ${ }^{54}$.

535 During the second surgical procedure we implanted intramuscular electrodes (Teflon-coated

536 stainless-steel wires, Cooner Wire, cat. no. AS631). Mk-Yg received electrodes in the following 537 arm and hand muscles: Deltoid (DEL), Biceps Brachii (BIC), Triceps Brachii (TRI), Extensor 538 Digitorium Communis (EDC), Flexor Carpi Radialis (FCR), Extensor Carpi Radialis (ECR), Flexor 539 Digitorium Superficialis (FDS). Mk-Br received an additional electrode in the Abductor Pollicis 540 Brevis (ABP). Due to practical constraints, Mk-Sa received electrodes only in Biceps Brachii (BIC), 541 Triceps Brachii (TRI) and Flexor Digitorium Superficialis (FDS). In all animals, wires were then 542 connected to an additional pedestal (Pedestal B), fixated to the titanium mesh.

543 During the third surgical procedure, monkeys were subjected to a lesion at the cervical level $(\mathrm{C} 5 / \mathrm{C} 6)$ of the spinal cord. The surgeon used a micro-blade to cut approximately one third of the dorsolateral aspect of the spinal cord, in order to interrupt the main component of the corticospinal tract unilaterally. All monkeys retained autonomic functions, as well as limited arm flexion and shoulder adduction capabilities. We monitored the animals for the first hours after surgery and several times daily during the following days. Monitoring scales (score sheets) were used to assess post-operative pain and general health condition during 1-2 weeks. Antibiotics were given immediately after the surgery and then once per day for 10 subsequent days, anti-inflammatory drugs were given once per day for 5 days (Rymadyl $4 \mathrm{mg} / \mathrm{kg}$, s.c.; Dexamethasone $0.3 \mathrm{mg} / \mathrm{kg}$, s.c.), and analgesic was given twice per day for 5 days (Temgesic $0.01 \mathrm{mg} / \mathrm{kg}$, i.m.). Within the same procedure, each monkey received a tailored epidural implant. The implant was inserted in the epidural space of the cervical spinal cord, according to methods described in Schiavone $2020^{57}$ and Capogrosso 201849. The implant was inserted below the T1 vertebra and pulled until it covered spinal segments from C6 to T1. We performed intra-operative electrophysiology in order to assess and refine the implant positioning so that electrodes are aligned to the animal-specific anatomical features. In particular, we verified that single pulses of stimulation delivered from the most rostral and most caudal electrodes elicited contractions in the BIC and FDS muscles respectively. We re-routed the wires subcutaneously in order to connect them to the Pedestal $B$. All surgical and post-operative care procedures were developed in details in previous reports ${ }^{49,50}$. For Mk-Sa, data presented in this paper were collected several weeks pre lesion and 1week post lesion, unfortunately a severe infection of the spinal array and EMGs that recurred after day 7 lead to the premature euthanasia of the monkey before the study could be completed in agreement with the endpoints in our veterinary authorization. For $\mathrm{Mk}-\mathrm{Br}$ and $\mathrm{Mk}-\mathrm{Yg}$ data presented in this paper were collected several weeks pre lesion and until 3 weeks post lesion. At the end of week 3 post lesion, Mk-Br had 2 episodes of self-mutilation on the foot ipsi-lateral to the lesion. In consequence we euthanized the animal before the end of the protocol according to the endpoints in our veterinary authorization. As described in the results section, we found postmortem that $\mathrm{Mk}-\mathrm{Br}$ had a medial spinal cord contusion at the T3 level. While this lesion did not affect motor control of the legs or the arms, it may have generated neuropathic pain. 
For Mk-Sa and Mk-Br, we acquired three-dimensional spatial coordinates of arm and hand joints using a 14-camera motion tracking system (Figure 1, Vicon Motion Systems, Oxford, UK) that tracked the Cartesian position of 6 infrared reflective markers (6 to $9 \mathrm{~mm}$ in diameter each, Vicon Motion Systems, Oxford, UK) at a $100 \mathrm{~Hz}$ framerate. All markers were placed on the left arm, one below the shoulder, three on the elbow (proximal, medial and distal position), and two on the left and right side of the wrist. For each subject, a model of the marker placement was calibrated in Vicon's Nexus software at the beginning of each experimental session. For Mk-Yg spatial coordinates of arm and hand joints were recorded using two cameras placed parallel to the sagittal and transversal plane of the animal (Vicon Motion Systems, Oxford, UK). The 3D coordinates of the arm and hand joints were extracted using DeepLabCut ${ }^{73}$. Due to the reduced informative content extracted from the camera parallel to the transverse plane, we then only used 2D coordinates on the animals' sagittal plane. The training set needed for automatic data labeling was created by manually labeling a subset of recorded videos. An investigator was blinded to the experimental condition and was instructed to mark four anatomical landmarks that mirrored the position of markers in Mk-Sa and $\mathrm{Mk}-\mathrm{Br}$ (shoulder, medial elbow, left and right wrist). Neural signals were acquired with a Neural Signal Processor (Blackrock Microsystems, USA) using the Cereplex-E headstage with a sampling frequency of $30 \mathrm{kHz}$. Electromyographic signals were acquired with a Behavioral Neurophysiology chronic recording system (RZ2 BioAmp Processor, Tucker-Davis Technologies, USA) at a sampling frequency of $12207 \mathrm{~Hz}$.

\section{Electrophysiology in sedated monkeys}

Monkeys were sedated with a continuous intravenous infusion of propofol $(5 \mathrm{ml} / \mathrm{kg} / \mathrm{h})$ that minimizes effects on spinal cord stimulation ${ }^{74}$. We delivered single pulses of cathodic, charge balanced, asymmetric square pulses $(0.3 \mathrm{~ms}, 1 \mathrm{~Hz})$ from each electrode contact while recording compound potentials from all implanted arm and hand muscles. Electromyographic signals were acquired with a Behavioral Neurophysiology chronic recording system (RZ2 BioAmp Processor, Tucker-Davis Technologies, USA) at a sampling frequency of $12207 \mathrm{~Hz}$. We then delivered 10 repetitions of pulse trains from each contact, at several frequencies ranging from 20 to $120 \mathrm{~Hz}$. We recorded compound potentials from all implanted arm and hand muscles and arm kinematics through two high resolution cameras (Sony FDR-X3000 Action Cam 4K). Through this procedure we identified three contacts that primarily elicited (1) arm flexors, (2) arm extensors and (3) hand flexors. In a reduced set of trials, we also recorded the force produced by arm flexion through a $10 \mathrm{~N}$ range force sensor (Dual-Range Force Sensor, DFS-BTA, Vernier, Beaverton, Oregon, USA). To record the pulling force produced during isometric arm flexion, the hand was fixated to the sensor hook through a string, and the sensor and the elbow were kept in place by two experimenters, in order to optimally capture the strength produced by muscle contraction.

\section{Behavioral experimental recordings}

All animals were trained to perform a three-dimensional robotic reach, grasp and pull task, previously described in detail in (Barra 201954) and briefly recalled here for simplicity. 
612 All animals were instructed to wait for a start signal by resting the left hand on a metallic bar. 613 When the "go-cue" was given, monkeys had to reach for and grasp a small spherical object 614 attached to the robot end effector and located in the three-dimensional space. The object was 615 placed approximately $180 \mathrm{~mm}$ above the animal seating height, $150 \mathrm{~mm}$ far from the 616 shoulder/head coronal plane and $30 \mathrm{~mm}$ left of the animal's left arm. Once animals got a hold on 617 the object, they had to pull it towards their own body until trespassing a virtual spatial threshold.

618 The accomplishment of such virtual threshold was automatically detected by the robot control 619 through online monitoring of the end effector position. Once attained the threshold, monkeys had 620 to let go on the object and go back to the metallic bar. Fruits and vegetables were used to reward 621 successful movements. Animals were trained daily (5 days per week) and every session ended 622 as soon as the animals showed any sign of fatigue or impatience.

\section{Stimulation during three-dimensional reach and pull task in injured monkeys}

All monkeys were recorded after injury as soon as they could independently move in their housing, feed themselves autonomously and did not show signs of discomfort. This corresponded to 3, 5 and 6 days after injury respectively for $\mathrm{Mk}-\mathrm{Yg}, \mathrm{Mk}-\mathrm{Br}$ and $\mathrm{Mk}-\mathrm{Sa}$. Each recording session was organized as follows. First, we recorded two blocks without stimulation, each of the duration of approximately 2 minutes. During those blocks we visually evaluated the impairment level of the animal and the performance of the brain decoder. Second, we used the brain decoder to trigger specific stimulation patterns. Contacts used to elicit those functions were defined through the experiments described in the previous paragraph and combined together to create stimulation protocols that allowed the animal to perform a full reach, grasp and pull movement.

635 We defined the movement performed by the animals as composed of three different phases: reach, grasp and pull. The identification of the reach phase was done by marking the moment in which the left hand left the metallic bar to when the hand closed around the object secured to the robot hand effector (the grasp event). The grasp phase was considered to be a window of 100 ms around the moment in which hand closed around the object. The pull phase started from the grasp event and finished when the animal accomplished the task by pulling the object across the virtual spatial threshold and placed the hand back on the resting bar. Events related to the 3 phases of the movement (movement onset: reaching, grasp onset: grasping and release of the object, and pulling) were identified manually by inspecting video recordings from Vicon Motion Systems (Oxford, UK). The same method was applied to mark successful and complete performance of reach, grasp and pull movements as events. A successful reach was defined as a complete extension of the arm that brought the hand at the position of the target (even when grasp could not be performed). A successful grasp was defined as a successful closure of the hand around the target. A successful pull was defined as the accomplishment of a complete flexion movement that brought the target past the virtual spatial threshold. Events were then 
650 extracted from Vicon and used to perform analysis on the kinematic of the movements and to 651 train the brain decoder by automatic routines (Matlab 2019b). All the analysis was conducted as

652 blinded experiments.

\section{Decoding motor states from intracortical signals}

654 We designed a neural decoder that detected reaching and grasping events using intracortical 655 spiking activity. In order to detect spikes, we set a threshold on each channel of -4 times the root656 mean-square voltage recorded during a brief period while the monkey was at rest. We estimated 657 firing rates in each of the motor cortical array channels by summing the multiunit spikes with a $658150 \mathrm{~ms}$ history every $0.5 \mathrm{~ms}$. We used these multiunit firing rate estimates to compute a twenty659 dimensional neural manifold capturing the majority of population variance ${ }^{64}$. We projected the 660 spiking activity onto this manifold to calibrate a multiclass regularized linear discriminant analysis 661 decoder 50 that predicted the labeled timing of reach and grasp events. The decoder used $500 \mathrm{~ms}$ 662 of past neural activity and output the probability of observing the reach and grasp events. During 663 calibration, we defined a probability threshold for each event ranging from 0.8 to 0.99 to optimize 664 predictions of the timing of each event using cross-validation. Since the monkeys could not 665 complete the task after $\mathrm{SCl}$, we were unable to consistently acquire labeled training data. We 666 therefore calibrated a decoding algorithm using reaches from a recording session of a healthy 667 monkey. We then manually labeled attempted reaches after SCI by manual inspection of video 668 recordings. Using canonical correlation analysis, we aligned the neural dynamics ${ }^{75}$ preceding 669 reaches on the healthy sessions to the observed neural dynamics preceding attempted reaches 670 after SCl. These aligned dynamics were used to control the decoder trained on the healthy 671 reaches.

672 We implemented a custom C++ software application running a control suite that used the 673 decoding algorithm to trigger EES stimulation in real-time. The application received neural data 674 over UDP and made predictions using the decoding algorithm at $15 \mathrm{~ms}$ intervals. When the output 675 probabilities crossed the defined threshold, the application triggered preprogrammed patterns of 676 EES.

\section{$677 \quad$ Analysis of muscle recruitment curves}

678 Electromyographic activity was bandpass filtered between 30 and $800 \mathrm{~Hz}$ with an offline $3^{\text {rd }}$ order 679 Butterworth filter and stimulus artifact were removed. For each animal, stimulation contact, muscle 680 and stimulation amplitude, we extracted compound potentials from 50ms-long segments of 681 electromyographic activity following a stimulation pulse. We then computed the peak-to-peak 682 amplitude of compound potentials. Since we gave four pulses of stimulation for each selected 683 current amplitude, we averaged across values corresponding to the same stimulation amplitude 684 and represented as the mean recruitment value of each muscle as a function of the injected 685 current. For each muscle, recruitment values have been subsequently normalized by the 686 maximum value obtained for that specific muscle, provided that we obtained response saturation 687 (and therefore maximal contraction) in at least one occasion during the session. In addition, we 
computed a selectivity index for each muscle ${ }^{76}$.

689 In order to obtain a comprehensive measure of muscle recruitment for each contact that would

690 allow to compare across animals, we computed, for each animal, each muscle and each contact, 691 an Average Recruitment Index (ARI) as the average of the recruitment values across all 692 stimulation amplitudes used from a specific stimulation site.

693 To compute muscle recruitment during the delivery of pulse train stimulation, we computed the 694 energy of the EMG signal during the duration of stimulation. We then applied the same 695 normalization procedure described above for single pulse recruitment.

\section{Analysis of muscle activity during EES}

697 Electromyographic activity was bandpass filtered between 30 and $800 \mathrm{~Hz}$ with an offline $3^{\text {rd }}$ order 698 Butterworth filter and stimulus artifact were removed. In all animals we computed the energy EMG 699 signals, for each implanted muscle. Energy of EMG signals during stimulation were computed on 700 each segment in which stimulation was delivered after the animal started a movement attempt. 701 Energy of EMG signals without stimulation were computed on each segment in which stimulation 702 was not delivered and the animal started a movement attempt. A movement attempt was defined 703 as an increased EMG activity of the Biceps and Deltoid muscles.

\section{Analysis of kinematics performance}

705

706

707

708

709

710

711

712

713

714

715

716

717

718

719

720

721

722

723

724

We performed Principal Component Analysis on a large set of kinematic features. We computed the features on data segments during the reach phase and the pull phase (see movement identification explained above, section Identification and classification of arm movements for kinematic analysis). All kinematic signals were previously low pass filtered at $6 \mathrm{~Hz}$. Segments were not interpolated nor resampled. Before performing PCA analysis, features were centered to have mean 0 and scaled to have standard deviation of 1 (Matlab 2019). The computed features for $\mathrm{Mk}-\mathrm{Br}$ included: minimum value, maximum value and total excursion of joint angles (shoulder flexion, elbow flexion, and wrist pronation); maximum, minimum and average angular velocity (for the shoulder flexion, elbow flexion and wrist pronation); minimum, maximum and average position along the sagittal, frontal and vertical axis of each arm joint (shoulder, elbow, wrist); maximum minimum and average wrist velocity along the sagittal, frontal and vertical axis; movement smoothness ${ }^{61}$; trajectory length during and time required to complete movements. All the listed features have been computed identically during the reach phase and the pull phase separately and treated as different features. In addition, computed maximal applied three-dimensional pulling force and the average position along the sagittal, frontal and vertical axis of each arm joint (shoulder, elbow, wrist) during grasp.

Since for Mk-Yg we only extracted 2D kinematics on the sagittal plane, the kinematic features for Mk-Yg included: minimum value, maximum value and total excursion of joint angles (shoulder flexion and elbow flexion); maximum and average angular velocity (for the shoulder flexion and elbow flexion); minimum, maximum and average position along the sagittal and vertical axis of 
each arm joint (shoulder, elbow, wrist); maximum and average wrist velocity along the sagittal and vertical axis; movement smoothness ${ }^{61}$; trajectory length during and time required to complete movements. All the listed features have been computed during the reach phase.

\section{Comparison of motor cortical activity during EES evoking movement and no movement}

To study how motor cortical activity interacted with EES, we analyzed the neural recordings from $\mathrm{Mk}-\mathrm{Br}$ and $\mathrm{Mk}-\mathrm{Yg}$. We identified periods where EES pulse trains produced no discernible movements by setting a threshold on hand velocity. We compared multi-unit neural firing rates on each channel in this period to neural firing rates in the previously identified trials where EES enabled reaching and grasping. First, we counted the number of spikes within the window of stimulation and divided by the duration of stimulation. We then averaged across stimulus repetitions of the movement and no movement conditions and pooled across recording sites in motor cortex.

We next computed instantaneous estimates of multi-unit firing rates on each channel by counting the number of spikes in non-overlapping $20 \mathrm{~ms}$ bins and convolving with a gaussian kernel of 50 ms width. We applied Principal Component Analysis (PCA) to compute 10-dimensional neural manifolds spanning this multi-unit population activity ${ }^{64}$. We projected the neural activity onto these manifold axes during the periods where EES evoked either movement or no movement. We then identified periods where the monkey was at rest with no EES, as well as periods where the monkey attempted movements of the arm with no EES. To compare the similarity of neural activity between these conditions, we computed the Mahalananobis distance between activity at rest and the three other periods: EES with movement, EES with no movement, and attempted movements with no EES.

\section{$\underline{\text { Histology }}$}

Monkeys were deeply anesthetized (lethal dose of pentobarbital, $60 \mathrm{mg} / \mathrm{kg}$, injected i.v.) and transcardially perfused with saline (about $200 \mathrm{ml}$ ), followed by 3 liters of $4 \%$ paraformaldehyde (PFA). Dissected spinal cord were post-fixed in 4\% PFA overnight, and then immersed in 30\% sucrose solution for 2 weeks. $50 \mu \mathrm{m}$ transverse or horizontal sections were cut using a cryostat and kept in $0.1 \mathrm{M}$ PBS azide $(0.03 \%)$ at $4^{\circ} \mathrm{C}$. Primary antibodies were: rabbit anti-lba1 (1:1000, Wako) and guinea pig anti-NeuN (1:300, Millipore). Fluorescence secondary antibodies were conjugated to: Alexa fluor 647 and Alexa fluor 555 (Life technologies). Sections were coverslipped using Mowiol. Immunofluorescence was imaged digitally using a slide scanner (Olympus VS-120). Lesions were reconstructed using image analysis software (Neurolucida) to trace the lesion over serial sections (200 $\mu \mathrm{m}$ apart).

\section{Statistical procedures}

All data are reported as mean values \pm standard error of the mean (s.e.m.) or mean values \pm 
761 standard deviation (std). The choice is highlighted directly in the figures or in the relative caption.

762 Significance was analyzed using the non-parametric Wilcoxon rank-sum test. In only one case

763 (Figure 5c), significance was analyzed using bootstrap. The level of significance was set at $764{ }^{*} p<0.05,{ }^{* *} p<0.01,{ }^{* * *} p<0.001$. 


\section{References}

1. ICCP. International Campaign for Cures of Spinal Cord Injury Paralysis. http://www.campaignforcure.org.

2. National Center for Chronic Disease Prevention and Health Promotion, Division for Heart Disease and Stroke. Stroke Facts. https://www.cdc.gov/stroke/facts.htm (2020).

3. Anderson, K. D. Targeting recovery: priorities of the spinal cord-injured population. Journal of neurotrauma 21, 1371-1383 (2004).

4. Moreland, J. D. et al. Needs assessment of individuals with stroke after discharge from hospital stratified by acute Functional Independence Measure score. Disability and rehabilitation 31, 2185-2195 (2009).

5. Lemon, R. N. Descending pathways in motor control. Annual review of neuroscience 31, 195-218 (2008).

6. Griffin, D. M., Hoffman, D. S. \& Strick, P. L. Corticomotoneuronal cells are 'functionally tuned'. Science 350, 667-70 (2015).

7. Griffin, D. M. \& Strick, P. L. The motor cortex uses active suppression to sculpt movement. Sci Adv 6, eabb8395 (2020).

8. Seki, K., Perlmutter, S. I. \& Fetz, E. E. Sensory input to primate spinal cord is presynaptically inhibited during voluntary movement. Nat Neurosci 6, 1309-16 (2003).

9. Lebedev, M. A. \& Nicolelis, M. A. Brain-machine interfaces: From basic science to neuroprostheses and neurorehabilitation. Physiological reviews (2017).

10. Ethier, C., Oby, E. R., Bauman, M. J. \& Miller, L. E. Restoration of grasp following paralysis through brain-controlled stimulation of muscles. Nature 485, 368-371 (2012).

11. Moritz, C. T., Perlmutter, S. I. \& Fetz, E. E. Direct control of paralysed muscles by cortical neurons. Nature 456, 639-642 (2008). 
792 12. Bouton, C. E. et al. Restoring cortical control of functional movement in a human with 793 quadriplegia. Nature (2016) doi:10.1038/nature17435.

794 13. Ajiboye, A. B. et al. Restoration of reaching and grasping movements through brain-

795 controlled muscle stimulation in a person with tetraplegia: a proof-of-concept demonstration.

796 The Lancet (2017) doi:10.1016/S0140-6736(17)30601-3.

797 14. Giat, Y., Mizrahi, J. \& Levy, M. A musculotendon model of the fatigue profiles of paralyzed 798 quadriceps muscle under FES. IEEE transactions on biomedical engineering 40, 664-674 799 (1993).

800 15. Popovic, M. R., Popovic, D. B. \& Keller, T. Neuroprostheses for grasping. Neurological $801 \quad$ research 24, 443-452 (2002).

802 16. Edgerton, V. R. et al. Training locomotor networks. Brain Res Rev 57, 241-254 (2008).

803 17. Holinski, B. J. et al. Intraspinal microstimulation produces over-ground walking in $804 \quad$ anesthetized cats. J Neural Eng 13, 056016 (2016).

805 18. Zimmermann, J. B., Seki, K. \& Jackson, A. Reanimating the arm and hand with intraspinal 806 microstimulation. Journal of neural engineering 8, 054001 (2011).

807 19. Gaunt, R. A., Prochazka, A., Mushahwar, V. K., Guevremont, L. \& Ellaway, P. H. Intraspinal 808 microstimulation excites multisegmental sensory afferents at lower stimulus levels than local 809 a-motoneuron responses. Journal of neurophysiology 96, 2995-3005 (2006).

810 20. Rattay, F., Minassian, K. \& Dimitrijevic, M. R. Epidural electrical stimulation of posterior 811 structures of the human lumbosacral cord: 2. quantitative analysis by computer modeling. $812 \quad$ Spinal cord 38, 473-489 (2000).

813 21. Capogrosso, M. et al. A computational model for epidural electrical stimulation of spinal 814 sensorimotor circuits. The Journal of neuroscience : the official journal of the Society for $815 \quad$ Neuroscience 33, 19326-40 (2013). 
22. Moraud, E. M. et al. Mechanisms Underlying the Neuromodulation of Spinal Circuits for

817 Correcting Gait and Balance Deficits after Spinal Cord Injury. Neuron 89, 814-28 (2016).

818 23. Formento, E. et al. Electrical spinal cord stimulation must preserve proprioception to enable

819 locomotion in humans with spinal cord injury. Nature neuroscience 21, 1728 (2018).

820 24. Wenger, N. et al. Closed-loop neuromodulation of spinal sensorimotor circuits controls

821 refined locomotion after complete spinal cord injury. Science translational medicine 6,

$822 \quad$ 255ra133-255ra133 (2014).

823 25. Harkema, S. et al. Effect of epidural stimulation of the lumbosacral spinal cord on voluntary 824 movement, standing, and assisted stepping after motor complete paraplegia: a case study.

$825 \quad$ The Lancet 377, 1938-1947 (2011).

826 26. Angeli, C. A., Edgerton, V. R., Gerasimenko, Y. P. \& Harkema, S. J. Altering spinal cord 827 excitability enables voluntary movements after chronic complete paralysis in humans. Brain

832 28. Courtine, G. et al. Transformation of nonfunctional spinal circuits into functional states after 833 the loss of brain input. Nat Neurosci 12, 1333-1342 (2009).

834 29. van den Brand, R. et al. Restoring Voluntary Control of Locomotion after Paralyzing Spinal $835 \quad$ Cord Injury. Science 336, 1182-1185 (2012).

836 30. Harkema, S. et al. Effect of epidural stimulation of the lumbosacral spinal cord on voluntary 837 movement, standing, and assisted stepping after motor complete paraplegia: a case study. $838 \quad$ Lancet 377, 1938-47 (2011).

839 31. Grahn, P. J. et al. Enabling Task-Specific Volitional Motor Functions via Spinal Cord $840 \quad$ Neuromodulation in a Human With Paraplegia. Mayo Clin Proc 92, 544-554 (2017). 
841 32. Angeli, C. A. et al. Recovery of Over-Ground Walking after Chronic Motor Complete Spinal

$842 \quad$ Cord Injury. N Engl J Med 379, 1244-1250 (2018).

843 33. Gill, M. L. et al. Neuromodulation of lumbosacral spinal networks enables independent

844 stepping after complete paraplegia. Nat Med (2018) doi:10.1038/s41591-018-0175-7.

845 34. Alam, M. et al. Evaluation of optimal electrode configurations for epidural spinal cord

846 stimulation in cervical spinal cord injured rats. Journal of neuroscience methods 247, 50-57

847 (2015).

848 35. Lu, D. C. et al. Engaging Cervical Spinal Cord Networks to Reenable Volitional Control of

849 Hand Function in Tetraplegic Patients. Neurorehabil Neural Repair 30, 951-962 (2016).

850 36. F. Inanici, L. N. Brighton, S. Samejima, C. P. Hofstetter, \& C. T. Moritz. Transcutaneous

851 spinal cord stimulation restores hand and arm function after spinal cord injury. IEEE

852 Transactions on Neural Systems and Rehabilitation Engineering 1-1 (2021)

853 doi:10.1109/TNSRE.2021.3049133.

854 37. Kapadia, N., Zivanovic, V. \& Popovic, M. Restoring voluntary grasping function in individuals

855 with incomplete chronic spinal cord injury: pilot study. Topics in spinal cord injury rehabilitation 19, 279-287 (2013).

857 38. Grillner, S. The motor infrastructure: from ion channels to neuronal networks. Nat Rev

$858 \quad$ Neurosci 4, 573-586 (2003).

859 39. McCrea, D. A. \& Rybak, I. A. Organization of mammalian locomotor rhythm and pattern 860 generation. Brain Res Rev 57, 134-46 (2008).

861 40. Bizzi, E., Giszter, S. F., Loeb, E., Mussa-Ivaldi, F. A. \& Saltiel, P. Modular organization of 862 motor behavior in the frog's spinal cord. Trends in neurosciences 18, 442-446 (1995).

863 41. Giszter, S. F. Motor primitives--new data and future questions. Curr Opin Neurobiol 33, $864 \quad 156-65(2015)$. 
42. Alstermark, B. \& Isa, T. Circuits for skilled reaching and grasping. Annu Rev Neurosci 35, 559-78 (2012).

43. Lemon, R. N. \& Griffiths, J. Comparing the function of the corticospinal system in different species: organizational differences for motor specialization? Muscle \& Nerve: Official Journal of the American Association of Electrodiagnostic Medicine 32, 261-279 (2005).

44. Kinoshita, M. et al. Genetic dissection of the circuit for hand dexterity in primates. Nature 487, 235-238 (2012).

45. Weiler, J., Gribble, P. L. \& Pruszynski, J. A. Spinal stretch reflexes support efficient hand control. Nat. Neurosci 1-11 (2019).

46. Omrani, M., Murnaghan, C. D., Pruszynski, J. A. \& Scott, S. H. Distributed task-specific

47. Sauerbrei, B. A. et al. Cortical pattern generation during dexterous movement is inputprocessing of somatosensory feedback for voluntary motor control. Elife 5, e13141 (2016). driven. Nature 577, 386-391 (2020).

48. Wenger, N. M. M., E; Gandar, J; Musienko, P; Capogrosso, M; Baud, L; Legoff, C; Barraud, Q; Pavlova, N; Domonici N; Minev, I; Asboth, L; Hirsch, A; Duis, S; Kreider, J; Mortera, A; Haverbeck, O; Kraus, S; Schmitz, F; DiGiovanna, J; van den Brand, R; Bloch, J; Detemple,

50. Capogrosso, M. et al. A brain-spine interface alleviating gait deficits after spinal cord injury P; Lacour, S; Bezard, E; Micera, S; Courtine G. Spatiotemporal neuromodulation therapies engaging muscle synergies to improve motor control after spinal cord injury. Nature Medicine (2015).

49. Capogrosso, M. et al. Configuration of electrical spinal cord stimulation through real-time processing of gait kinematics. Nat Protoc (2018) doi:10.1038/s41596-018-0030-9. in primates. Nature 539, 284-288 (2016).

51. Wagner, F. B. et al. Targeted neurotechnology restores walking in humans with spinal cord injury. Nature 563, 65 (2018). 
52. Ladenbauer, J., Minassian, K., Hofstoetter, U. S., Dimitrijevic, M. R. \& Rattay, F. Stimulation of the Human Lumbar Spinal Cord With Implanted and Surface Electrodes: A Computer Simulation Study. Neural Systems and Rehabilitation Engineering, IEEE Transactions on 18, 637-645 (2010).

53. Greiner, N. et al. Recruitment of Upper-Limb Motoneurons with Epidural Electrical Stimulation of the Primate Cervical Spinal Cord. Nat Commun (2021).

54. Barra, B. et al. A Versatile Robotic Platform for the Design of Natural, Three-Dimensional Reaching and Grasping Tasks in Monkeys. Journal of Neural Engineering (2019) doi:10.1088/1741-2552/ab4c77.

55. Jenny, A. B. \& Inukai, J. Principles of motor organization of the monkey cervical spinal cord. J Neurosci 3, 567-75 (1983).

56. Capogrosso, M. et al. A brain-spine interface alleviating gait deficits after spinal cord injury in primates. Nature 539, 284-288 (2016).

57. Schiavone, G. et al. Soft, Implantable Bioelectronic Interfaces for Translational Research. Advanced Materials 32, 1906512 (2020).

58. Chao, Z. C., Sawada, M., Isa, T. \& Nishimura, Y. Dynamic Reorganization of Motor Networks During Recovery from Partial Spinal Cord Injury in Monkeys. Cereb Cortex (2018) doi:10.1093/cercor/bhy172.

59. Freund, P. et al. Nogo-A-specific antibody treatment enhances sprouting and functional recovery after cervical lesion in adult primates. Nature medicine 12, 790-792 (2006).

60. Sharpe, A. N. \& Jackson, A. Upper-limb muscle responses to epidural, subdural and intraspinal stimulation of the cervical spinal cord. J Neural Eng 11, 016005 (2014).

61. Teulings, H.-L., Contreras-Vidal, J. L., Stelmach, G. E. \& Adler, C. H. Parkinsonism

913 Reduces Coordination of Fingers, Wrist, and Arm in Fine Motor Control. Experimental

$914 \quad$ Neurology 146, 159-170 (1997). 
915 62. Hofstoetter, U. S. et al. Periodic modulation of repetitively elicited monosynaptic reflexes of

916 the human lumbosacral spinal cord. Journal of neurophysiology jn 001362015 (2015)

917 doi:10.1152/jn.00136.2015.

918 63. Gerasimenko, Y. P. et al. Spinal cord reflexes induced by epidural spinal cord stimulation in 919 normal awake rats. Journal of Neuroscience Methods 157, 253-263 (10).

920 64. Gallego, J. A., Perich, M. G., Miller, L. E. \& Solla, S. A. Neural Manifolds for the Control of $921 \quad$ Movement. Neuron 94, 978-984 (2017).

922 65. Lempka, S. F. et al. Patient-specific analysis of neural activation during spinal cord 923 stimulation for pain. Neuromodulation: Technology at the Neural Interface 23, 572-581 924 (2020).

925 66. Schirmer, C. M. et al. Heuristic map of myotomal innervation in humans using direct 926 intraoperative nerve root stimulation. J Neurosurg Spine 15, 64-70 (2011).

927 67. Moritz, C. T., Lucas, T. H., Perlmutter, S. I. \& Fetz, E. E. Forelimb movements and muscle 928 responses evoked by microstimulation of cervical spinal cord in sedated monkeys. Journal 929 of neurophysiology 97, 110-120 (2007).

930 68. Kato, K., Nishihara, Y. \& Nishimura, Y. Stimulus outputs induced by subdural electrodes on 931 the cervical spinal cord in monkeys. Journal of Neural Engineering 17, 016044 (2020).

932 69. Song, S. \& Geyer, H. A neural circuitry that emphasizes spinal feedback generates diverse 933 behaviours of human locomotion. The Journal of physiology (2015) doi:10.1113/JP270228.

934 70. Confais, J., Kim, G., Tomatsu, S., Takei, T. \& Seki, K. Nerve-specific input modulation to 935 spinal neurons during a motor task in the monkey. Journal of Neuroscience 37, 2612-2626 936 (2017).

937 71. Ting, J. et al. A wearable neural interface for detecting and decoding attempted hand 938 movements in a person with tetraplegia. in 1930-1933 (IEEE, 2019). 
939 72. National Research Council (US) Institute for Laboratory Animal Research. Guide for the

$940 \quad$ Care and Use of Laboratory Animals. (National Academies Press (US), 1996).

941 73. Mathis, A. et al. DeepLabCut: markerless pose estimation of user-defined body parts with

942 deep learning. Nature neuroscience 21, 1281-1289 (2018).

943 74. Toossi, A. et al. Effect of anesthesia on motor responses evoked by spinal neural

944 prostheses during intraoperative procedures. Journal of neural engineering 16, 036003

945 (2019).

946 75. Gallego, J. A., Perich, M. G., Chowdhury, R. H., Solla, S. A. \& Miller, L. E. Long-term

947 stability of cortical population dynamics underlying consistent behavior. Nature

948 neuroscience 23, 260-270 (2020).

949 76. Raspopovic, S., Capogrosso, M. \& Micera, S. A Computational Model for the Stimulation of

950 Rat Sciatic Nerve Using a Transverse Intrafascicular Multichannel Electrode. IEEE

951 Transactions on Neural Systems and Rehabilitation Engineering 19, 333-344 (2011). 


\section{Figures}

\section{A Experimental setup}

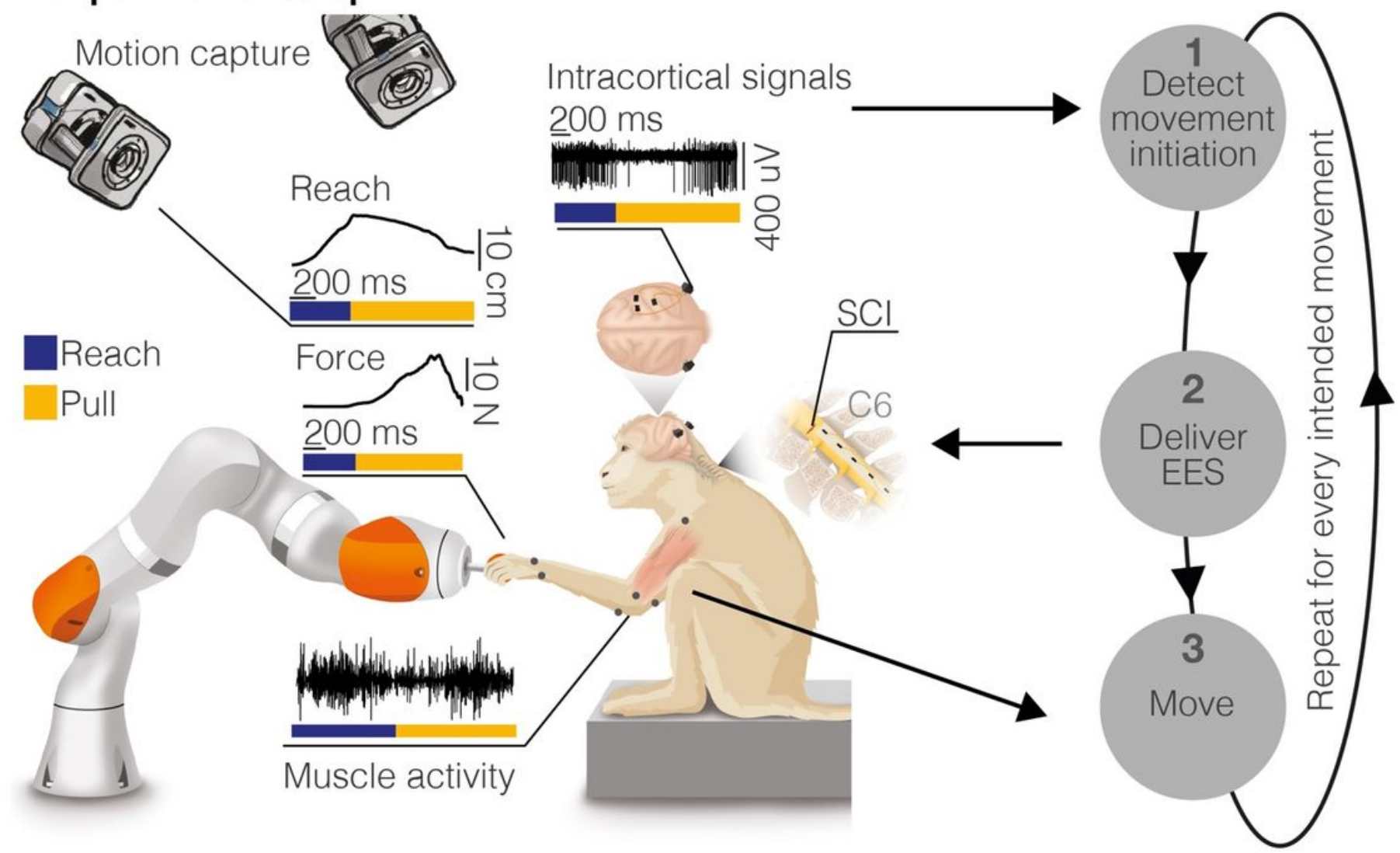

\section{B Behavioral task}

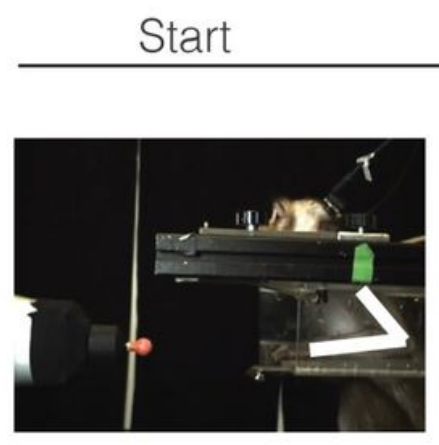

\section{REACH}
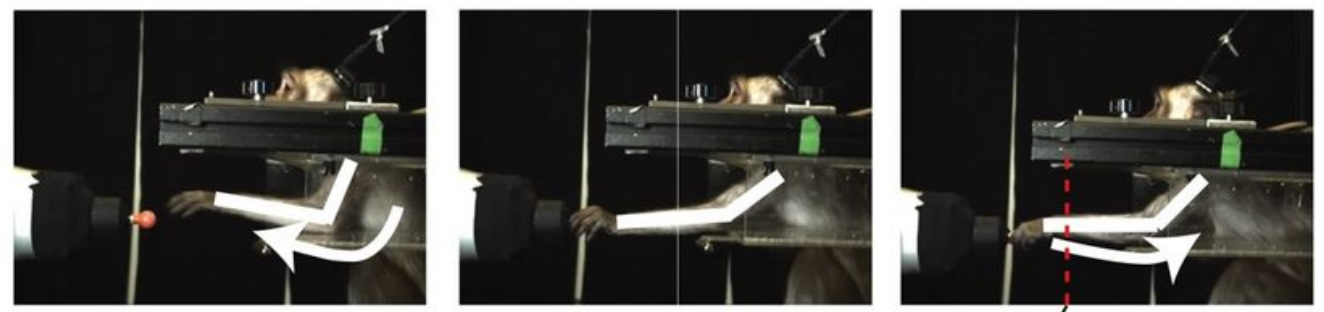

Task completed when passing threshold

Figure 1

Experimental framework. (A) On the left, schematic of the behavioral experimental platform. While the animals were performing a robotic reach, grasp and pull task, we measured 3D forces applied to the robot joints, full-limb kinematics, electromyographic (EMG) activity from eight muscles of the arm and hand, and intra-cortical signals from sensorimotor areas. On the right, conceptual scheme of the experimental protocol: (1) A decoder running on a control computer identified movement attempts and (2) delivered electrical spinal cord stimulations to the appropriate spinal roots. (3) Stimulations produced arm and 
hand movement that we recorded and analyzed off-line. (B) Schematic illustration of the task. Monkeys were trained to reach for, grasp, and pull a target object placed at the end effector of a robotic arm. We considered a movement complete when a target spatial threshold was crossed during pull. Copyright Jemère Ruby.

A

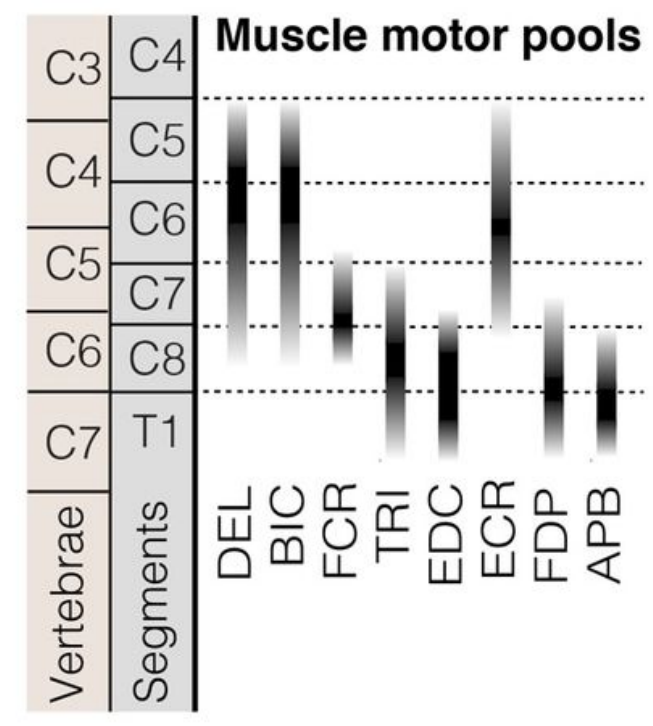

B Anatomical landmarks

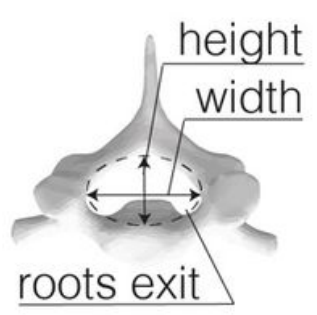

C5-T1 Length

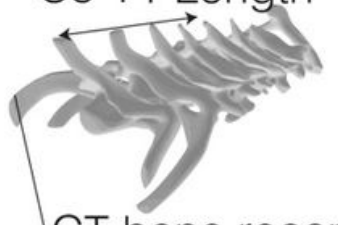

CT bone reconstruction
Personalization landmarks

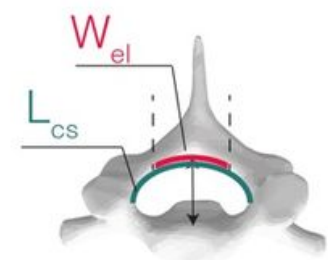

$W_{\text {el }}=1 / 3 L_{c s}$

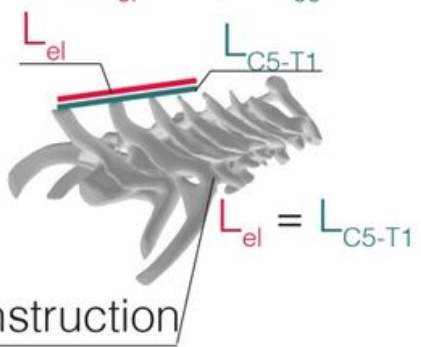

C Electrode implantation

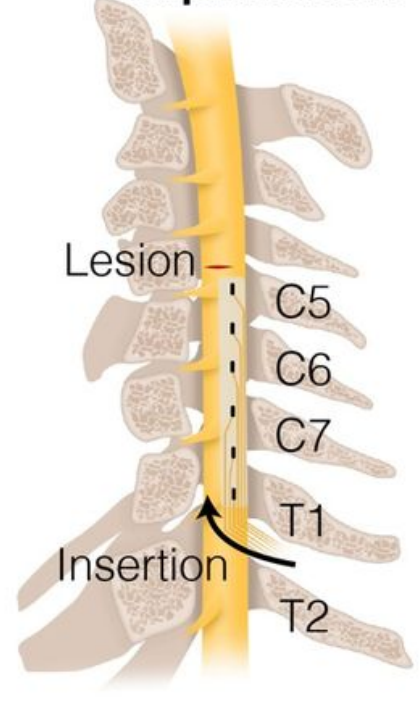

D

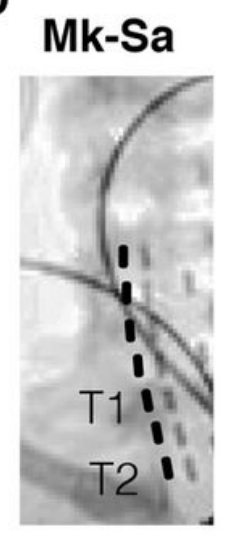

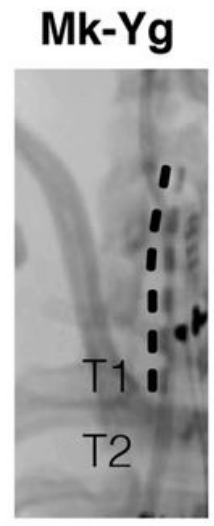

E
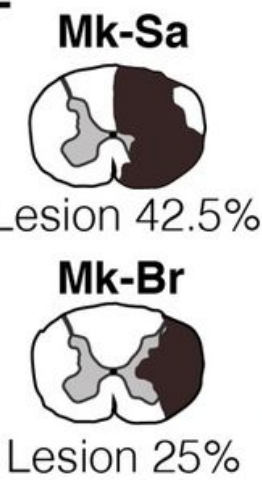

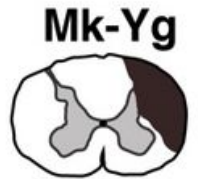

Lesion $19,5 \%$

Grey M.

$\square$ White M.

Lesion

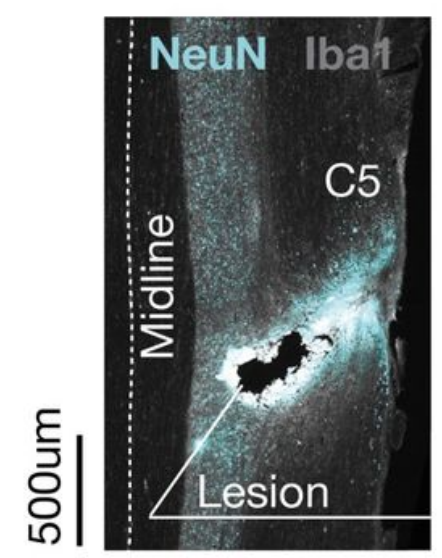

Figure 2

Epidural electrode design and implantation. (A) Motoneurons pool distribution of arm and hand muscles in the cervical spinal cord in relation to vertebrae and spinal segments (adapted from Jenny and Inukai, 1983). Deltoid (DEL), Biceps Brachii (BIC), Flexor Carpi Radialis (FCR), Triceps Brachii (TRI), Extensor Digitorium Communis (EDC), Extensor Carpi Radialis (ECR), Flexor Digitorium Profundis (FDP), Abductor Pollicis Brevis (ABP). (B) Anatomical landmarks used to tailor the epidural interface to each monkey's anatomy (Length of dorsal aspect of spinal canal Lcs, length of C5-T1 spinal segment LC5-T1, electrode width Wel, electrode length Lel). Three-dimensional reconstructions of vertebras are obtained by CTreconstruction (Osirix, Pixmeo, Switzerland). (C) Schematic representation illustrating the positioning and insertion of the spinal implant in the epidural space (D) Representative Xray scans of the epidural implant in the three monkeys (Mk-Sa, Mk-Br and Mk-Yg). (E) Anatomical reconstruction of the cervical spinal cord 
lesion (black area) for the 3 monkeys, shown on a transversal section (the percentage indicates the portion of the total spinal cord area that was injured on this transversal plane). On the right, representative image of longitudinal section of the spinal cord of $\mathrm{Mk}-\mathrm{Br}$ around the lesion site stained with NeuN (neuronal cell bodies) and Iba1 (microglia).

A Muscle recruitment during single pulse

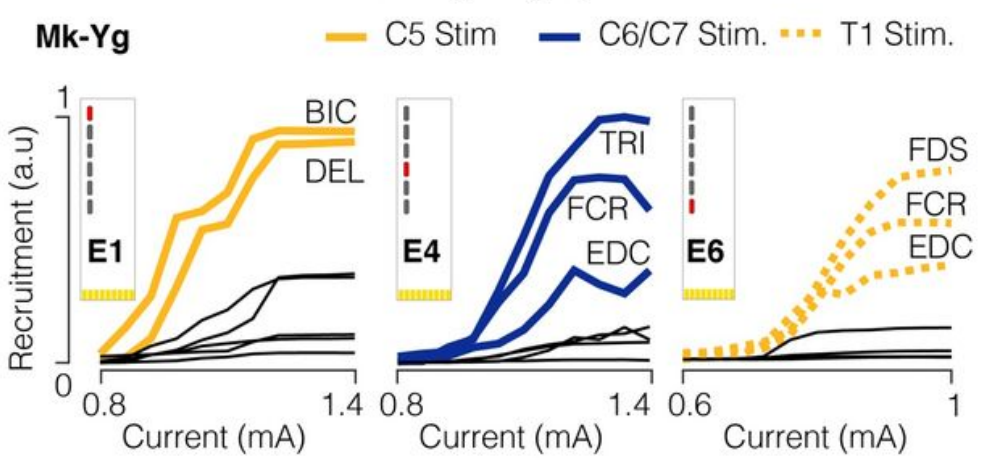

B Similar muscle recruitment in three monkeys

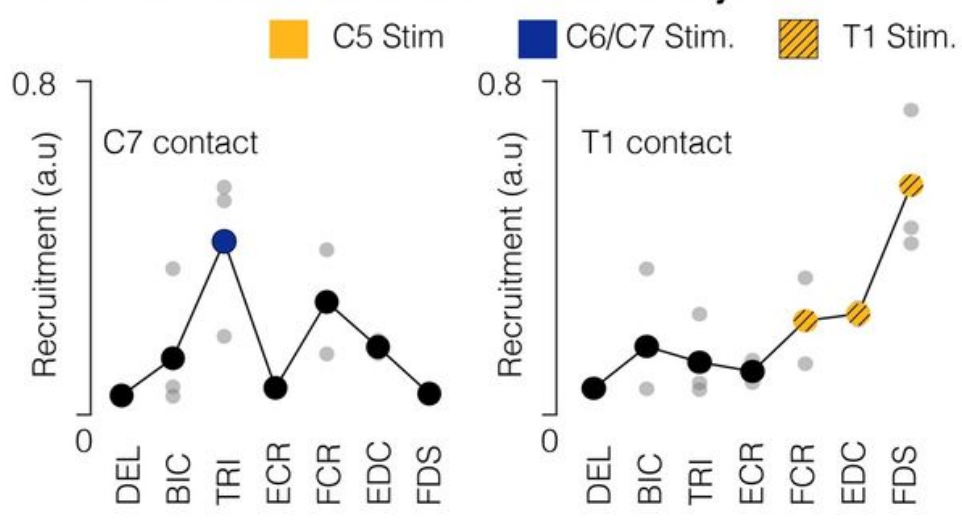

C Muscle recruitment during pulse trains
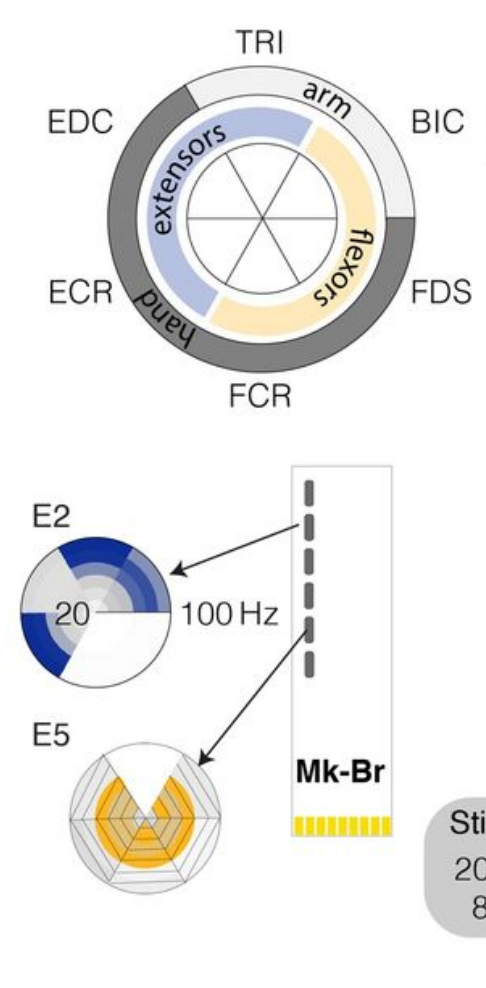

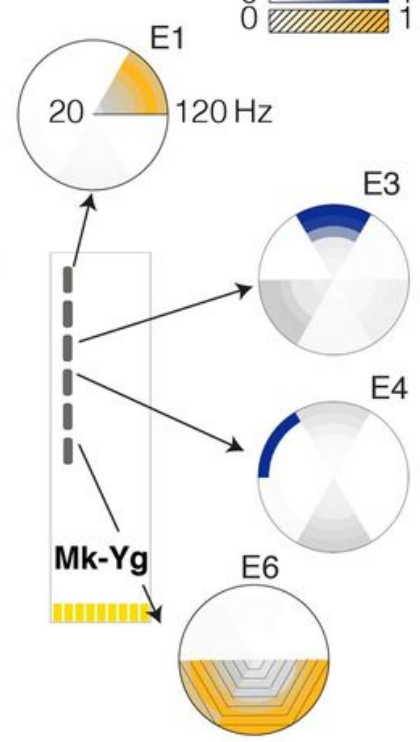

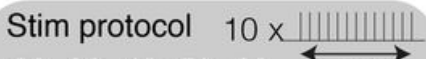
$20,30,40,50,60, \longleftrightarrow 1 \mathrm{~s}$ $80,100,120 \mathrm{~Hz}$

\section{Figure 3}

Muscle recruitment of spinal stimulation. (A) Examples of muscle recruitment obtained by stimulating (1 $\mathrm{Hz}$ ) at $\mathrm{C} 5, \mathrm{C} 6 / \mathrm{C} 7$, and $\mathrm{T} 1$ spinal segments (Mk-Yg). (B) Average muscle activations elicited from $\mathrm{C} 7$ and $T 1$ contacts in $n=3$ monkeys (grey bullets: for each animal, average recruitment across all stimulation currents. Big bullets: mean of average recruitments across animals). (C) Muscle recruitment obtained during delivery of pulse trains in anesthetized monkeys. Recruitment was estimated by computing the energy of EMG signals for each muscle and each stimulation contact. Stimulation frequencies ranged from 20 to $120 \mathrm{~Hz}(n=2)$. For each muscle, energy values were normalized to the maximum value obtained across all frequencies and contacts. 


\section{Arm movement is modulated by stimulation frequency}

Mk-Yg

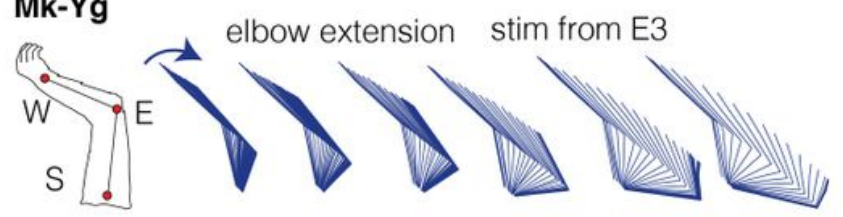

wrist flexion stim from E6

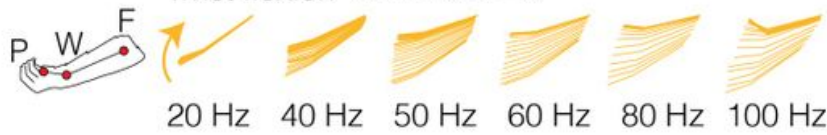

$\mathrm{Mk}-\mathrm{Br}$

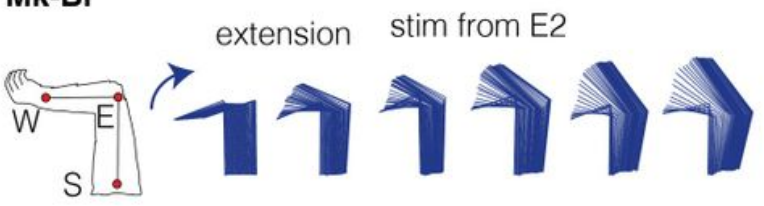

Mk-Yg

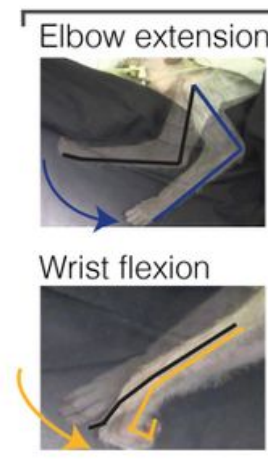

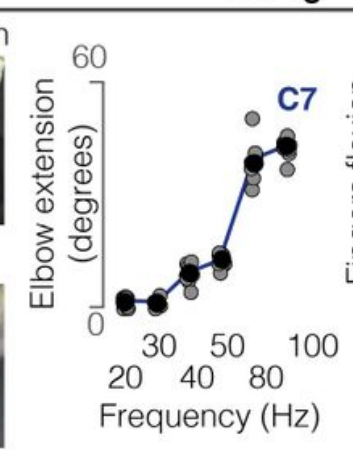

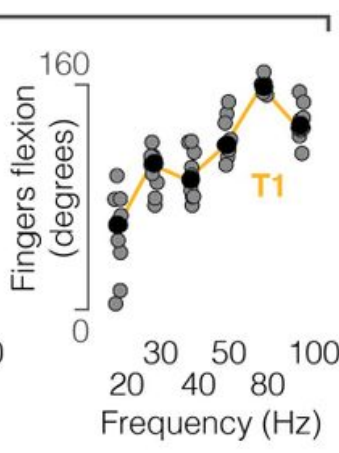

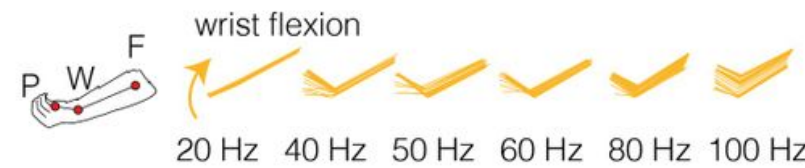

$\mathrm{Mk}-\mathrm{Br}$

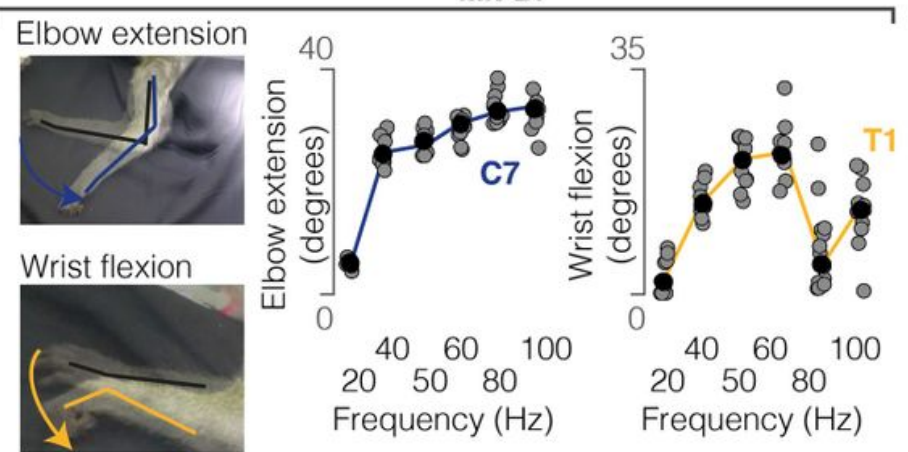

Figure 4

EES produces single joint movements in anesthetized animals. Top: stick diagram schematic of movements elicited by pulse-trains of stimulation in anesthetized conditions. Mk-Br: on the left, arm kinematic obtained by delivering stimulation at different frequencies from contacts number 2 and 5 (counting from the top); for $\mathrm{Mk}-\mathrm{Yg}$, on the right, arm kinematic obtained by delivering stimulation at different frequencies from contacts number 3 and 6 . Bottom: single joint angles excursions induced by stimulation at C7 (blue) and T1 (yellow) roots. Stimulation frequencies ranged from 20 to $100 \mathrm{~Hz}(\mathrm{n}=2)$. Black bullets: mean. Line: interpolation of the mean values. 


\section{A Frequency selection}

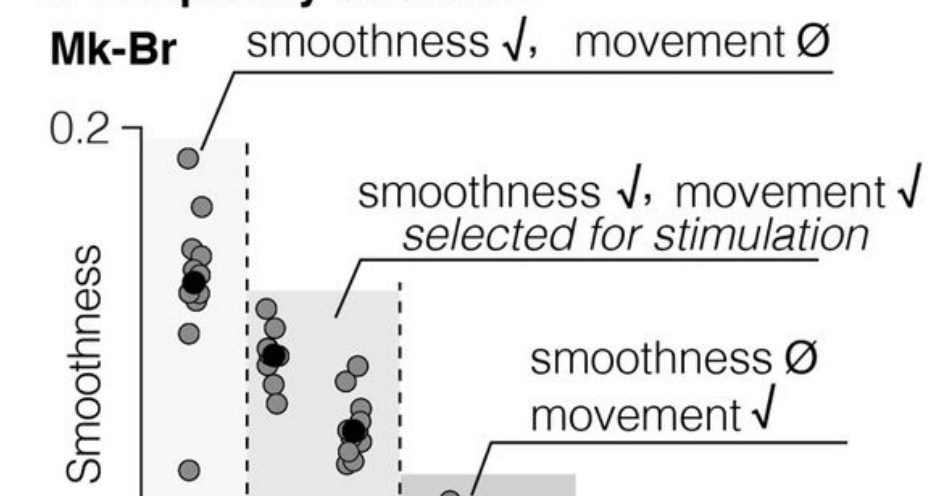

B Electrode selection

Reach Grasp

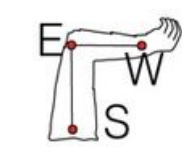

elbow extension

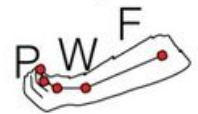

hand and wrist flexion

Pull

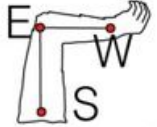

elbow

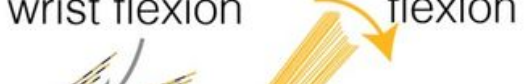

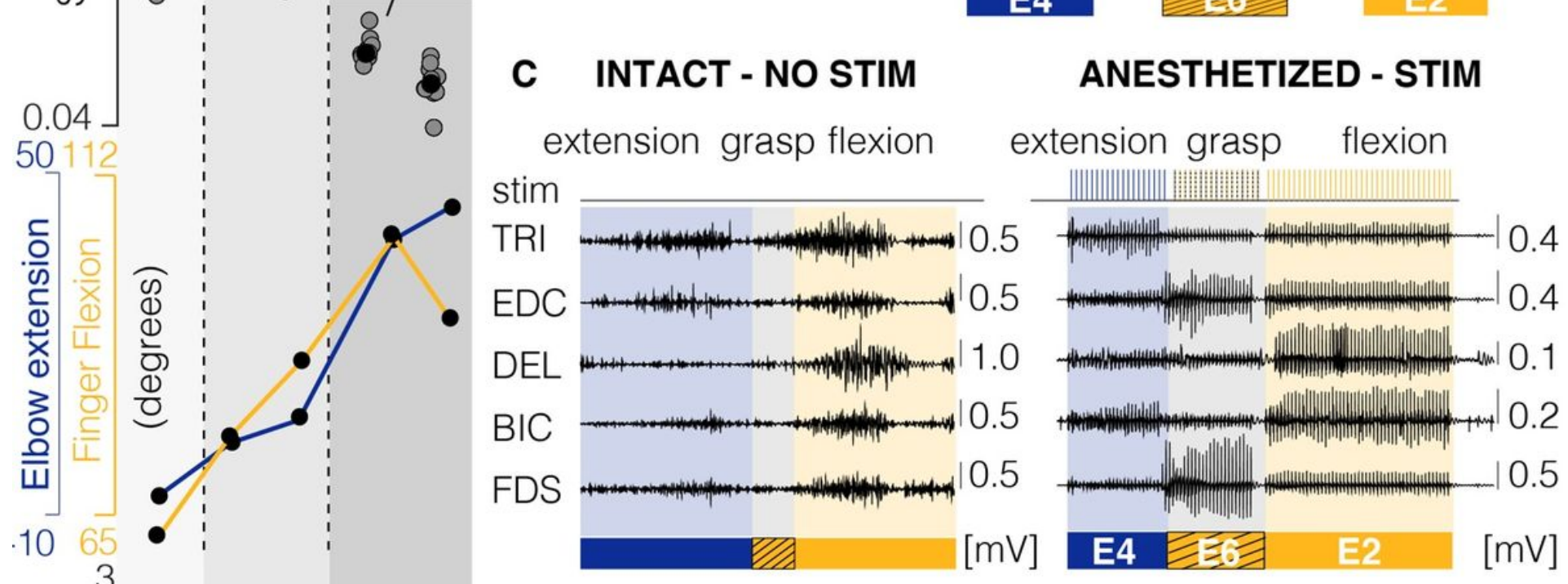

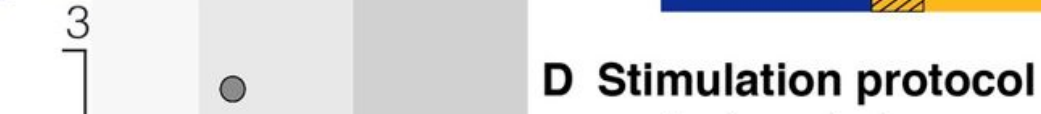

D Stimulation protocol
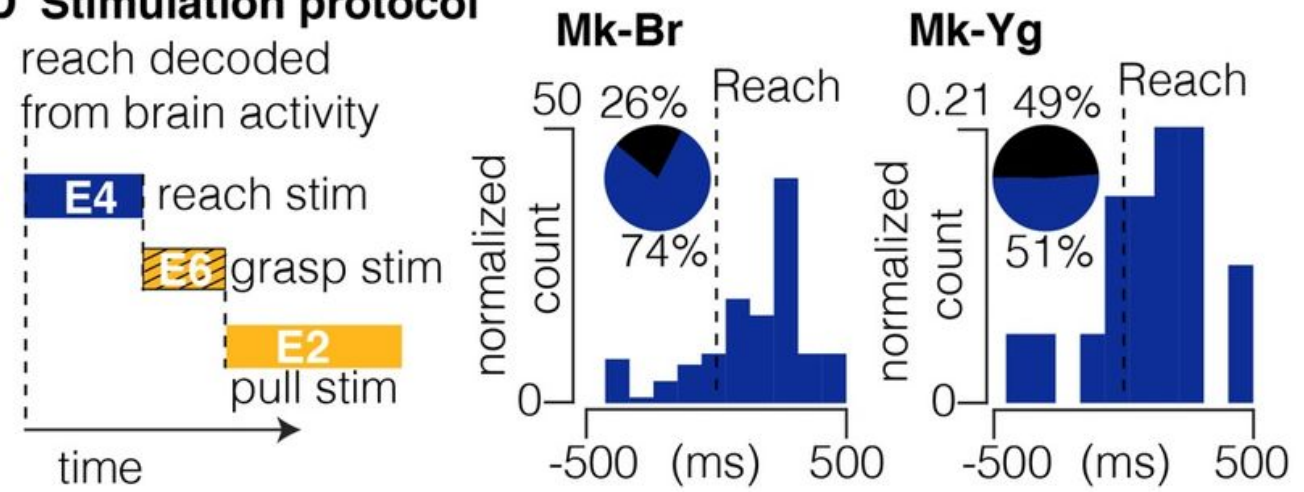

Figure 5

Design of stimulation protocol. (A) Combined representation of movement smoothness, elbow and finger flexion, and pulling force during anesthetized stimulation. Shades of gray highlight three frequency ranges that produce: (1) smooth trajectory, but little movement and low force $(2 \mathrm{~Hz}),(2)$ smooth trajectory, extended movement and medium force $(40$ and $50 \mathrm{~Hz}),(3)$ abrupt and very extended movement and low force $(80$ and $100 \mathrm{~Hz}$ ). The range $40-50 \mathrm{~Hz}$ was selected as the best optimization of sufficient movement, smoothness and force production. (B) Schematic representation of arm and hand kinematics during stimulation delivered from the selection of three contacts to produce elbow extension (blue), hand and wrist flexion (yellow and black), and elbow flexion (yellow). (C) Example of comparison between EMG activity during intact movement (left) and movement elicited by chaining stimulation from the three 
selected contacts (right). (D) Scheme illustrating how stimulation is triggered from movement-related intra-cortical signals. On the right, online performances of movement attempt decoder in two animals with SCl. Pie charts represent percentage of predicted (blue) and unpredicted (black) reach events by our decoder.

A

Intact

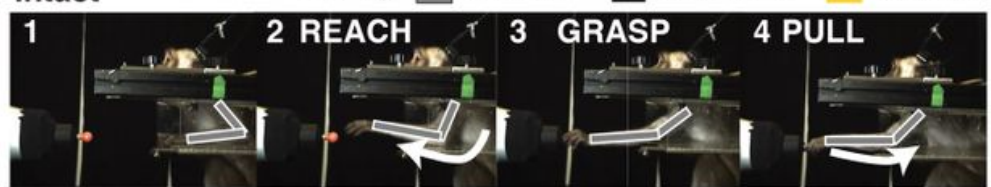

After $\mathrm{SCl}$ - stim OFF

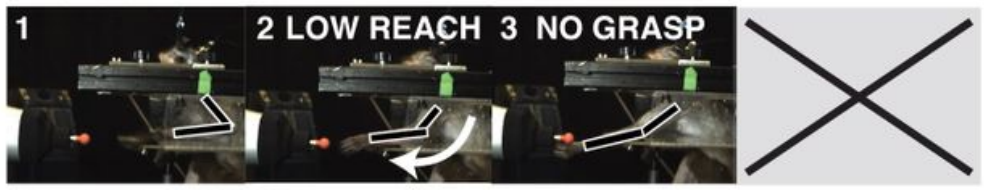

After SCl - stim ON

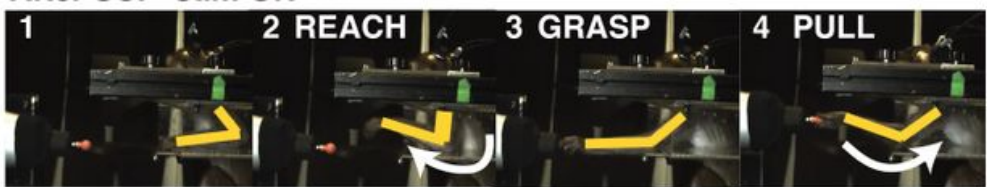

C EES improves task performance Mk-Br, week 3

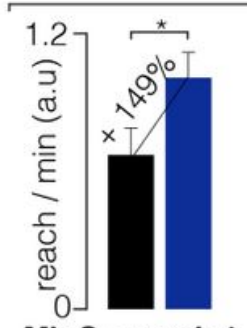

Mk-Sa, week 1
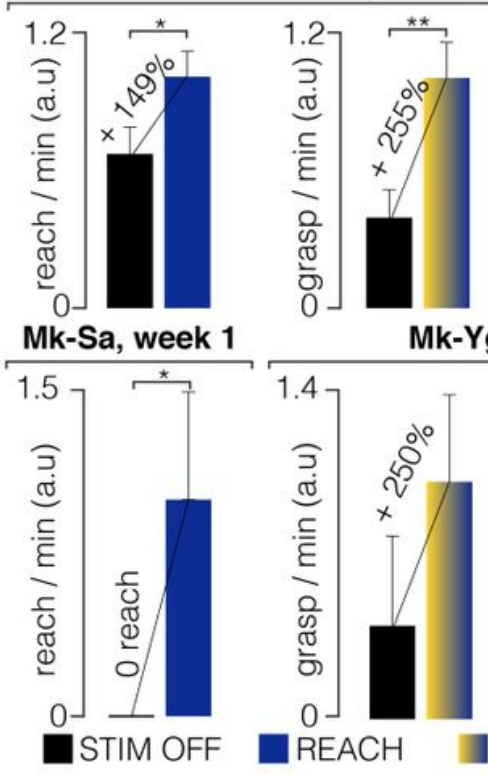

Mk-Yg, week 2

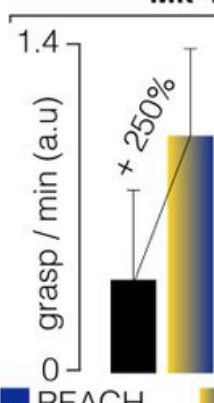

$\mathrm{REACH}$
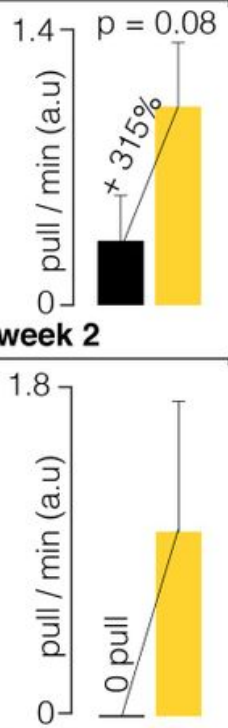

D EES improves kinematics

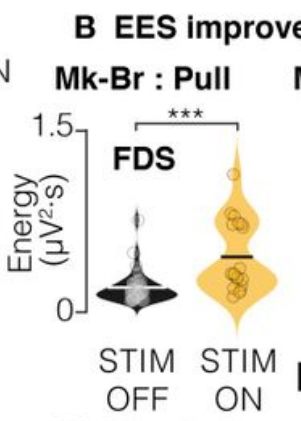

Mk-Yg : Pull

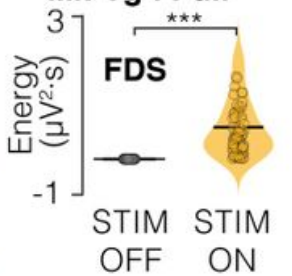

Mk-Br STIM OFF STIM ON

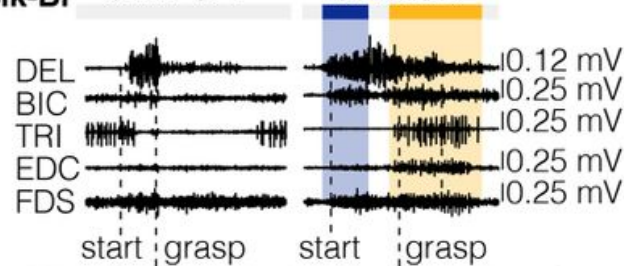

STIM OFF —REACH STIM —PULL STIM

Mk-Br : Reach
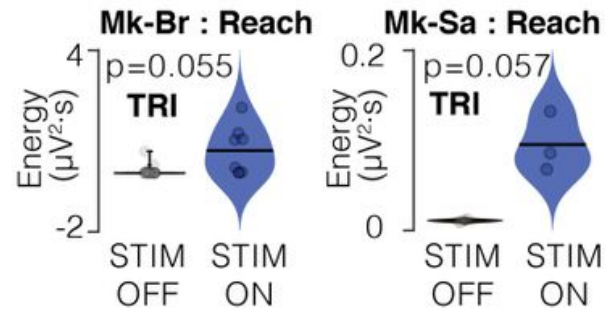

Mk-Yg
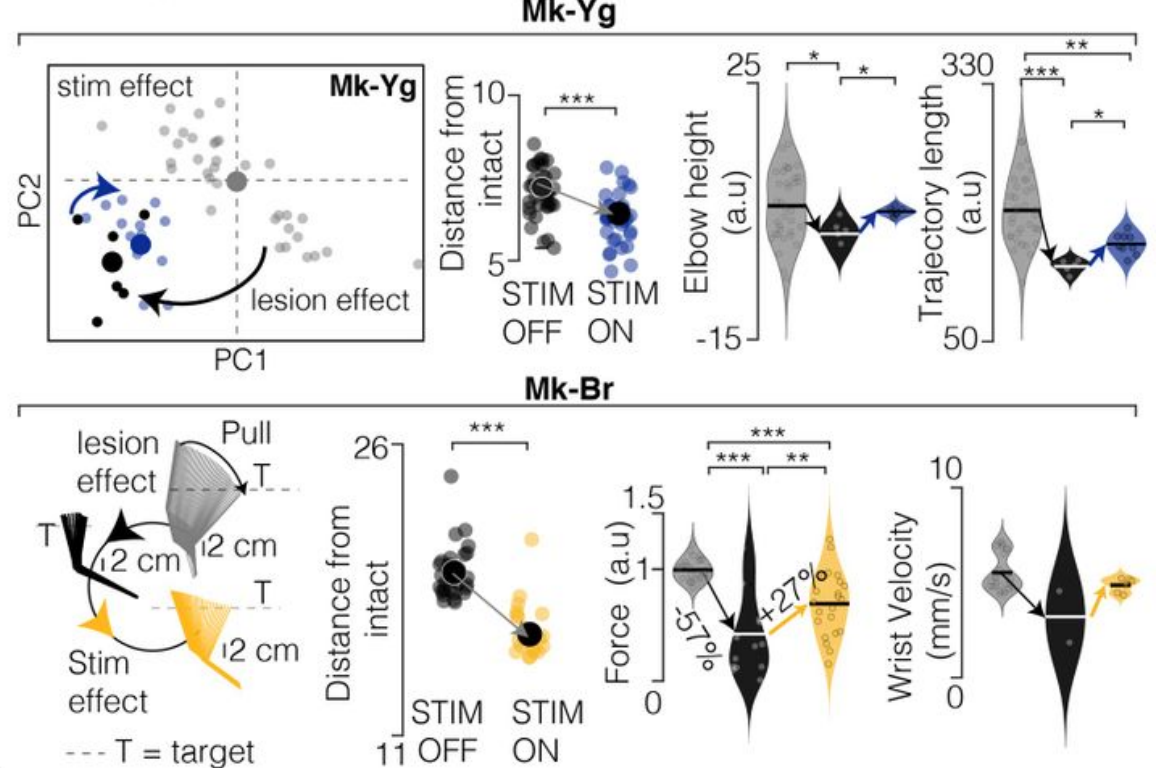

$\mathrm{Mk}-\mathrm{Br}$

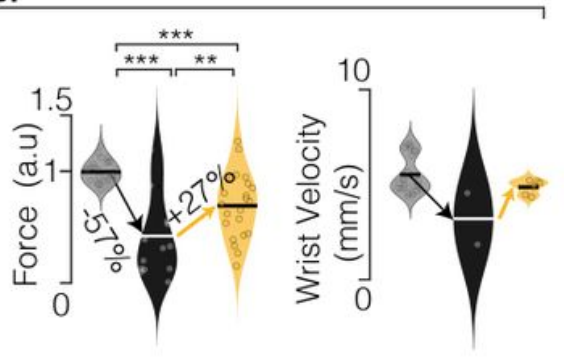

\section{Figure 6}

EES improves task performance, muscle strength and movement quality. (A) Snapshots of Mk-Yg performing the task before $\mathrm{SCl}$, after $\mathrm{SCl}$ without $\mathrm{EES}$, and after $\mathrm{SCl}$ with EES. A full successful trial is composed of a reach, a grasp, and a pull. After $\mathrm{SCl}, \mathrm{Mk}-\mathrm{Yg}$ could only perform reaching movements without EES, while when EES was delivered the full task could be performed. (B) Violin plots of signal energy of triceps and FDS EMG profiles during reach ( $\mathrm{Mk}-\mathrm{Br}$ and $\mathrm{Mk}-\mathrm{Sa}$ ) and pull (Mk-Br and $\mathrm{Mk}-\mathrm{Yg}$ ). All individual data points are represented by bullets. Black lines correspond to the mean of the distribution. Statistical analysis with Wilcoxon Ranksum test. On the right, example raw EMG data after SCl with and without EES. (C) Bar plots report the rate of successful movements after $\mathrm{SCl}$, without and with 
stimulation. Data are presented as mean \pm STD and normalized on the mean value in stimulation condition. Statistics was performed with Bootstrap. (D) Example PC analysis of kinematic features (See methods). Top-left, first and second PC space. Bottom left, stick diagram representation of arm kinematics during pull in intact conditions, after SCl without and with EES. At the immediate right (both bottom and top), euclidean distance in the feature space of trials without stimulation (black) and with stimulation (blue) from the centroid of the trials in intact condition. At the extreme right, example violin plots of movement quality features in the three conditions: intact, after $\mathrm{SCl}$, and after $\mathrm{SCl}$ with stimulation. Statistics with Wilcoxon Ranksum test. Asterisks: ${ }^{\star} p<0.05,{ }^{\star \star} p<0.01,{ }^{\star \star *} p<0.001$. 
A

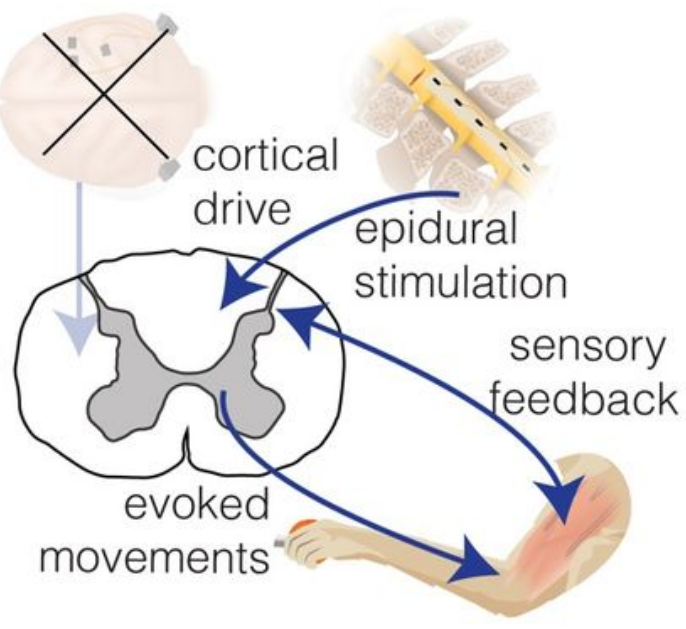

B
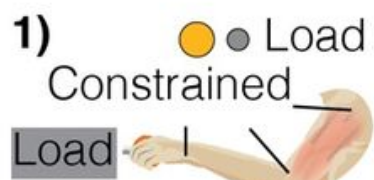

2) $-\circ \mathrm{No}$ load Unconstrained

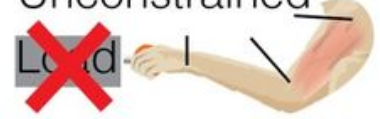

\section{Biceps}

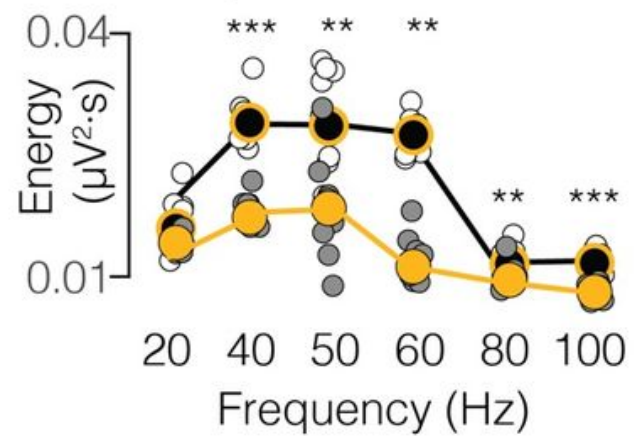

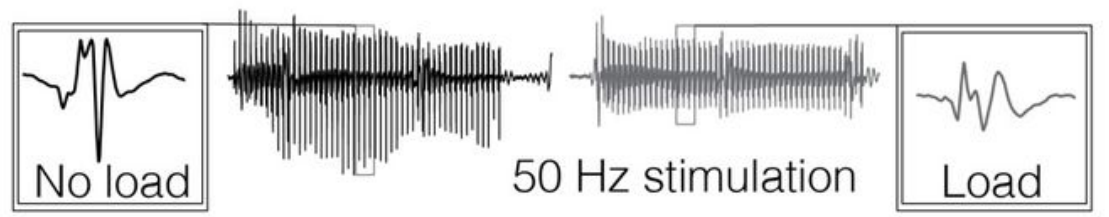

\section{Stimulation is gated by cortical drive}

C

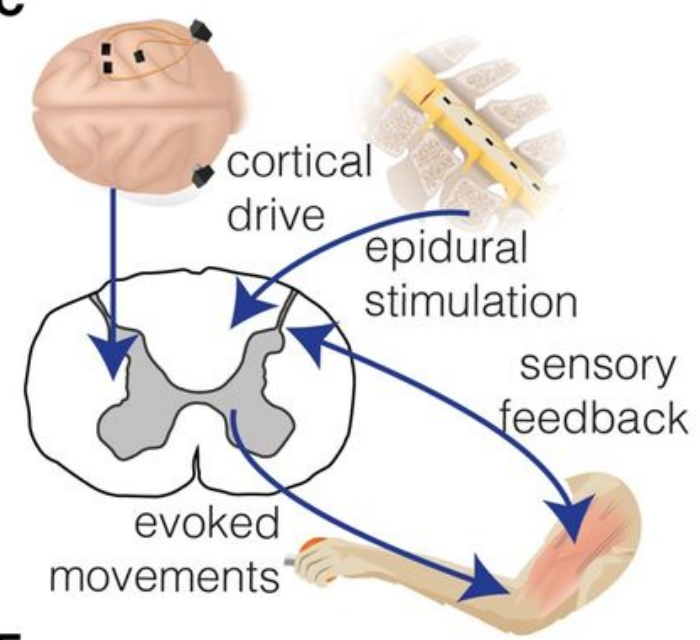

E

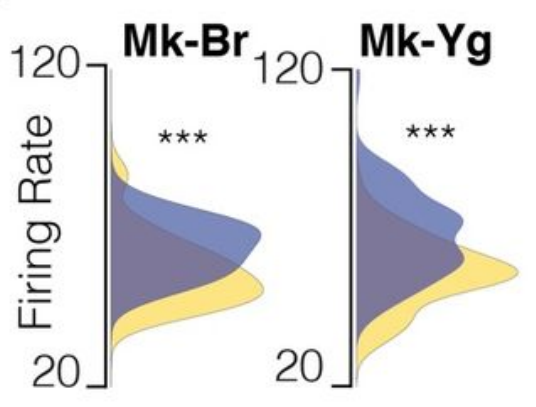

D

$\mathrm{Mk}-\mathrm{Br}$

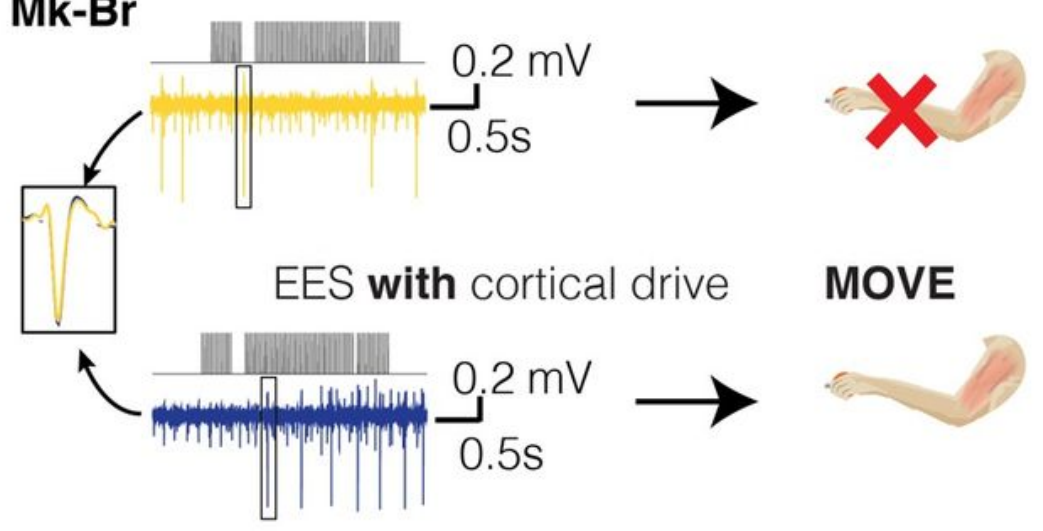

$\mathbf{F}$

$\mathrm{Mk}-\mathrm{Br}$

- Rest

- No Move

- Move

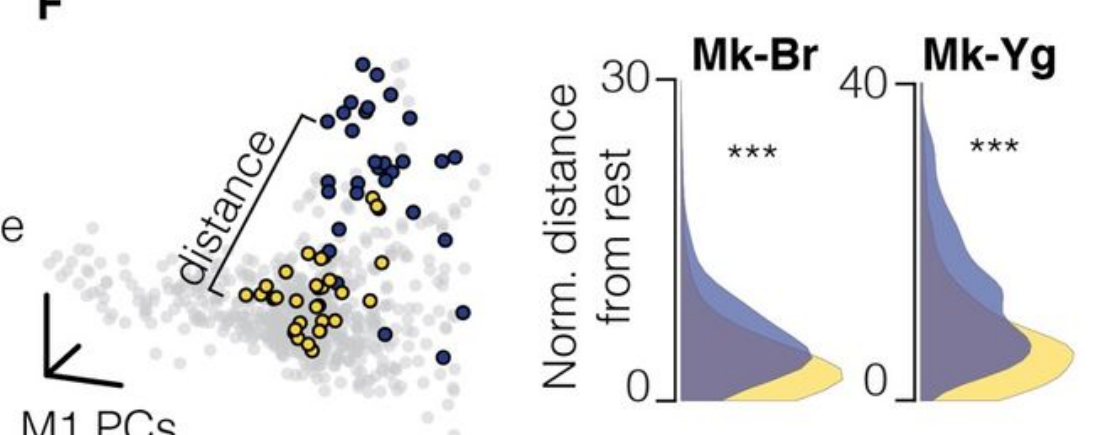

Figure 7

EES must be synchronized with motor intention. (A) Schematic of the interactions between EES and residual neural structures during anesthetized stimulation. During anesthesia, cortical control has no interaction, therefore EES interacts solely with sensory feedback spinal circuits. (B) Quantification of EMG activity during EES in two conditions: unconstrained arm (no load, black); arm constrained by load applied at the hand (load, gray). White and grey bullets: individual data points for no load and load 
conditions. Black and yellow bullets: mean values for no load and load conditions. Black and yellow lines: interpolation of mean values for no load and load conditions. On the bottom, example of EMG traces obtained during stimulation in the no-load (black) and load (gray) conditions. Stimulation artifacts have been removed. (C) Schematic of interactions between EES and residual neural structures during the performance of the behavioral task. EES interacts with descending cortical drive sent through residual pathways after $\mathrm{SCl}$, as well as with sensory spinal circuits. (D) Schematic illustrating the kinematic outcome of the interaction between EES and residual cortical inputs. The same EES pulse train (top) applied to Mk-Br can result in different motor outputs: no movement output when the cortex is silent (yellow, top), movement is produced when the cortex is active (blue, bottom). (E) Distribution of average firing rates across all $\mathrm{M} 1$ channels during stimulation trains that evoked no movement (yellow) and movement (blue). (F) Left: State space view of M1 activity for all time points during rest (gray), successful stimulation (blue) and unsuccessful stimulation (yellow). The brain states during unsuccessful stimulation (yellow) overlapped with the rest states, while the successful stimulation (blue) did not. Right: we computed a relative Mahalanobis distance between the two stimulation conditions and the cluster of neural states at rest. For both monkeys, neural states during stimulation periods with no movement were close to rest.

\section{Supplementary Files}

This is a list of supplementary files associated with this preprint. Click to download.

- Video1.mp4

- Video5.mp4

- SupplementaryData.pdf

- Video4.mp4

- Video3.mp4

- Video2.mp4

- SupplementaryData.pdf 